This item was submitted to Loughborough's Research Repository by the author.

Items in Figshare are protected by copyright, with all rights reserved, unless otherwise indicated.

\title{
Structure formation in the oceanic subsurface bubble layer by an internal wave field
}

PLEASE CITE THE PUBLISHED VERSION

http://dx.doi.org/10.1063/1.3499379

PUBLISHER

(C) American Institute of Physics

VERSION

VoR (Version of Record)

LICENCE

CC BY-NC-ND 4.0

\section{REPOSITORY RECORD}

Grimshaw, Roger H.J., Karima R. Khusnutdinova, L.A. Ostrovsky, and A.S. Topolnikov. 2019. "Structure Formation in the Oceanic Subsurface Bubble Layer by an Internal Wave Field". figshare.

https://hdl.handle.net/2134/15727. 


\section{AIP $\mid$ Physics of Fluids}

\section{Structure formation in the oceanic subsurface bubble layer by an internal wave field}

R. H. J. Grimshaw, K. R. Khusnutdinova, L. A. Ostrovsky, and A. S. Topolnikov

Citation: Physics of Fluids (1994-present) 22, 106603 (2010); doi: 10.1063/1.3499379

View online: http://dx.doi.org/10.1063/1.3499379

View Table of Contents: http://scitation.aip.org/content/aip/journal/pof2/22/10?ver=pdfcov

Published by the AIP Publishing

\section{Articles you may be interested in}

Evidence of Doppler-shifted Bragg scattering in the vertical plane by ocean surface waves

J. Acoust. Soc. Am. 131, 2011 (2012); 10.1121/1.3683247

Generation of intermediately long sea waves by weakly sheared winds

Phys. Fluids 23, 016604 (2011); 10.1063/1.3549622

Numerical investigation of rising bubble wake and shape variations

Phys. Fluids 21, 122102 (2009); 10.1063/1.3271146

Simulation of micro gas bubble generation of uniform diameter in an ultrasonic field by a boundary element method

Phys. Fluids 18, 108102 (2006); 10.1063/1.2364139

Laboratory images of breaking internal waves

Phys. Fluids 18, 091107 (2006); 10.1063/1.2335963

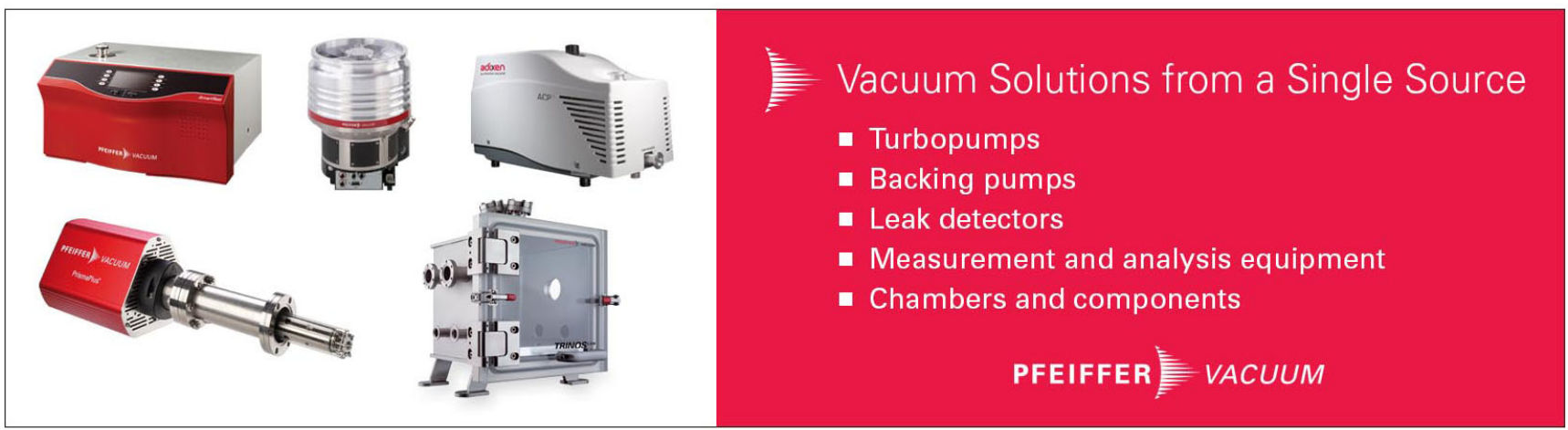




\title{
Structure formation in the oceanic subsurface bubble layer by an internal wave field
}

\author{
R. H. J. Grimshaw, ${ }^{1}$ K. R. Khusnutdinova, ${ }^{1, a)}$ L. A. Ostrovsky, ${ }^{2}$ and A. S. Topolnikov ${ }^{3}$ \\ ${ }^{1}$ Department of Mathematical Sciences, Loughborough University, Loughborough LE11 3TU, \\ United Kingdom \\ ${ }^{2}$ Zel Technologies/NoAA Earth System Research Laboratory and University of Colorado, 325 Broadway St., \\ Boulder, Colorado 80305-3337, USA \\ ${ }^{3}$ Institute of Mechanics, Ufa Branch of the Russian Academy of Sciences, \\ 71 Prospect Oktyabrya, Ufa 450054, Russia
}

(Received 7 March 2010; accepted 30 August 2010; published online 27 October 2010)

\begin{abstract}
We model the effects of an internal wave on the structure of the oceanic subsurface bubble layer, generated by breaking surface waves. We consider two situations: when breaking is caused either by a strong sustained wind or by the direct interaction of surface waves with an internal wave. We find that the effects are twofold; bubbles are driven by the internal wave field and the injection of bubbles into the water is enhanced in downwelling areas behind the crests of the internal wave. We use an uncoupled problem formulation, substituting the solution for an internal wave in a two-layer fluid model into the equations describing the bubble dynamics. The latter equations are solved numerically, showing structure formation in the bubble layer for each of the two cases, when one of the aforementioned mechanisms dominates the other. (c) 2010 American Institute of Physics.

[doi:10.1063/1.3499379]
\end{abstract}

\section{INTRODUCTION}

It is well-known that breaking surface waves injects bubbles into the subsurface layer of the ocean. For winds exceeding $6.5-7 \mathrm{~m} / \mathrm{s}$, there is a continuous bubble layer of variable thickness, which may extend to a depth of several meters beneath the surface. ${ }^{1-5}$ The observed bubble layer is highly structured, varies both spatially and temporally, and depends significantly on wind speed. In general, there is a monotonic decrease in the bubble void fraction with increasing depth. The void fraction profile either decays exponentially, with a typical $e$-folding scale of order $1 \mathrm{~m}$, which depends on the wind speed (see Ref. 6) or it may follow an inverse-square profile. ${ }^{7}$ The volume-scaled representation of the number density of bubbles as a function of the bubble radius has a peak at all depths and decreases rapidly on either side of the peak and also rapidly with depth (see, e.g., Refs. 5 and 8-10 and the references therein). These observations have shown that bubbles with radii of approximately 50-100 $\mu \mathrm{m}$ contribute most to the total void fraction, except very close to the surface. ${ }^{5}$

Recent observations of the structure of the bubble layer (e.g., Refs. 2, 5, and 10) and developments in the mechanics of multiphase media (e.g., Refs. 11-14), have allowed us to begin the consideration of the effect of bubbles on internal waves. ${ }^{15-17}$ Our results indicated that bubble distributions, when present, can have a profound effect on the structure of the internal wave field (but do not significantly affect the surface wave mode). Bubbles can support their own "bubble" modes of internal waves, even in an otherwise

\footnotetext{
${ }^{a)}$ Author to whom correspondence should be addressed. Electronic mail: k.khusnutdinova@lboro.ac.uk.
}

homogeneous fluid. If there is a background density stratification which is present in the absence of any bubbles, supporting one or more internal modes, then the weak coupling introduced through the interaction of the two waveguides induces a splitting of the dispersion curves, which is reflected in the behavior of the respective modal functions. In the simple case when the background density stratification (in the absence of bubbles) is modeled by a two-layer fluid so that there is just a single pycnocline mode, the splitting of the dispersion curves is most pronounced for a shallow pycnocline and a relatively large void fraction for the bubbles compared to the density jump across the interface. Otherwise, for a deeper pycnocline, for a larger density jump across the pycnocline, or for a smaller void fractions of bubbles, the splitting of the dispersion curves is still present, but is less pronounced, resulting in a pycnocline mode which has a similar structure to the bubble modes. Finally, if the pycnocline is well separated from the bubble layer, then there is virtually no interaction between the pycnocline mode and the bubble modes and we have two independent waveguides located at different depths.

This present study is devoted to the opposite problem of the effects that an internal wave might have on the subsurface bubble layer. There are some observations of such effects in the ocean, ${ }^{18-20}$ which motivate this study. We consider an uncoupled problem formulation, where we look at the dynamics of a bubble layer in the presence of a specified internal wave field, modeled as an interfacial wave in a twolayer fluid. This representation for the internal wave field is substituted into the equation describing the bubble dynamics (similar to Refs. 2 and 7-9 in the studies of the steady-state distributions), which are then solved numerically to reveal the structure formation in the bubble layer. We consider two 


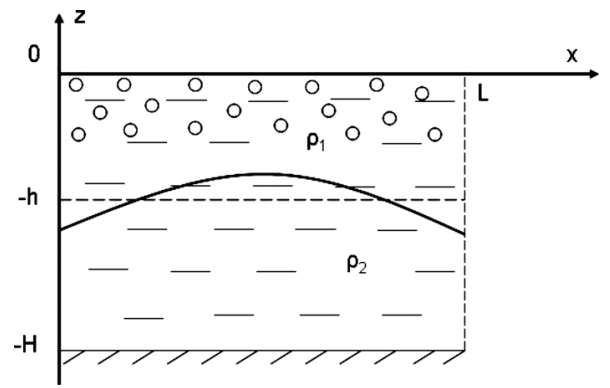

FIG. 1. Problem formulation: A two-layer fluid with a subsurface bubble layer.

cases. In the first case, the bubbles are injected by breaking surface waves generated by a strong wind and this is represented by a source term which is spatially homogeneous in the horizontal direction. In the second case, the surface waves are directly modulated by the internal wave, leading to a source of bubbles which is spatially inhomogeneous in the horizontal direction; here we adapt and develop an approach of Baschek et al. ${ }^{21}$ to model this situation. Since bubbles significantly affect the acoustic properties of a fluid, we illustrate some consequences of the described physical effects by calculating, along with hydrodynamic characteristics such as the bubble void fraction, the acoustic wave speed in the mixture and the scattering intensity. Some relevant acoustic data are available in Refs. 19 and 20.

Our paper is set out as follows. Section II describes the problem formulation used to model the structure formation in the bubble layer in the presence of an internal wave. The numerical scheme is described in Sec. III. In Sec. IV, we first consider the case when the bubble layer is supported by a sustained strong wind when we assume that the source of bubbles from the surface on average is homogeneous in the horizontal direction, while it is decaying in the vertical direction. In Sec. V, we consider a second case, when the wind is weak, and instead the bubble layer is supported by the interaction of surface waves with an internal wave propagating along a shallow pycnocline; the bubble source term is now highly inhomogeneous in the horizontal direction, as well as decaying in the vertical direction. We conclude in Sec. VI, comparing the qualitative predictions of our study with some existing observations. The solution for an internal wave in a two-layer fluid is briefly overviewed in Appendix A. A summary of some theoretical results related to the steepening of surface waves due to their interaction with an internal wave is given in Appendix B.

\section{PROBLEM FORMULATION}

The internal wave field constitutes a fundamental component of the ocean and is a subject of active research (for example, see Refs. 22-25). For sufficiently large horizontal scales, it is customary to reduce the study of internal waves to a normal mode structure with associated horizontal propagation features (e.g., Refs. 26-28).

In this paper, we aim to model the structure formation in the bubble layer due to an internal wave propagating along a sharp pycnocline (see Fig. 1). We consider a twodimensional problem formulation and use the spatial coordinates $(x, z)$, where $x$ is horizontal and $z$ is vertical. The undisturbed free surface is at $z=0$. We use the well-known model of a two-layer fluid (see Appendix A), where we have just one pycnocline mode in the absence of any bubbles. Note that this situation changes if we also take account of the depth-dependent bubble distribution when we have infinitely many new modes supported by the bubbles (see Ref. 17).

To describe the temporal and spatial concentration of bubbles in the fluid we extend the work of Thorpe ${ }^{2,8,9}$ and Buckingham $^{7}$ and use the model

$$
\begin{aligned}
\frac{\partial N}{\partial t} & +\frac{\partial}{\partial x}(N u)+\frac{\partial}{\partial z}(N v) \\
& =\frac{\partial}{\partial x}\left(K_{v} \frac{\partial N}{\partial x}\right)+\frac{\partial}{\partial z}\left(K_{v} \frac{\partial N}{\partial z}\right)-\sigma_{*} N+q(x, z, t) .
\end{aligned}
$$

Here, $N$ is the number density of bubbles, $K_{v}$ is the turbulent diffusion coefficient, $\sigma_{*}$ is the dissolution rate (assumed to be constant), $q(x, z, t)$ is the source of bubbles injected through the surface, $u$ is the horizontal velocity of bubbles, which coincides with the horizontal velocity of the fluid [given approximately by formulae (A7) and (A10) in Appendix A], and $v$ is the vertical velocity of bubbles, related to the vertical velocity of the fluid [given approximately by formulae (A8) and (A11) in Appendix A] by

$$
v=w+v_{\infty},
$$

where, following Ref. 8, we use the relations

$$
v_{\infty}=\frac{2}{9} \frac{a^{2} g \rho_{l}}{\mu_{l}}\left[\left(y^{2}+2 y\right)^{1 / 2}-y\right], \quad y=10.82 \frac{\mu_{l}^{2}}{\rho_{l}^{2} g a^{3}}
$$

for the rise speed of bubbles with respect to the fluid at equilibrium. Here, $\mu_{l}$ is the coefficient of dynamic viscosity of the fluid, $a$ is the bubble radius, and $g$ is gravity. Equation (1) describes the evolution of $N$ in time and space caused by convection [second and third terms in the left hand side of Eq. (1)], turbulent diffusion (first and second terms in the right-hand side), dissolution $\sigma_{*} N$, and source $q(x, z, t)$.

In Eq. (1), $q(x, z, t)$ is a number density of bubbles injected through the surface to any depth in unit time. When the bubble layer is supported by a strong sustained wind, we will model the source of bubbles by a horizontally and temporally homogeneous source in a thin surface layer, which we represent simply as $q_{0} \delta(z+\epsilon), 0<\epsilon \ll H$ (see Sec. IV). However, this situation becomes invalid in the case when breaking is caused primarily by the steepening of surface waves due to interaction with an internal wave, propagating close to the sea surface. Indeed, in this case we can no longer assume the bubble layer to be horizontally and temporally homogeneous. In Sec. V, we find an expression for the source term, following Ref. 21. In both cases, the source term is confined to a very thin layer near the surface when compared to the resulting depth-dependent bubble distribution and it is then convenient to replace the source term in Eq. (1) with a surface flux condition 


$$
\left.\left(\frac{K_{v}}{N} \frac{\partial N}{\partial z}-v_{\infty}\right) N\right|_{z=0}=\int_{-H}^{0} q(x, z, t) d z
$$

For the case of a bubble layer supported by a strong sustained wind, when $q(x, z, t)=q_{0} \delta(z+\epsilon)$, the right-hand side of Eq. (2) reduces to just $q_{0}$. Since $N$ quickly decreases with depth, the bottom condition does not affect the solution noticeably; here we use the zero flux $\partial N / \partial z=0$ condition at $z=-H$.

Let us suppose that the majority of bubbles have almost equal size, which depends on values of $x, z$, and $t$, i.e., $a=a(x, z, t)$. The bubble radius can be found from the equation for the change of the bubble mass due to dissolution (similar to Ref. 2)

$$
\frac{d}{d t_{b}}\left(\frac{4}{3} \pi \rho_{g} a^{3}\right)=-4 \pi a D \kappa\left(p_{g}-p\right) \mathrm{Nu},
$$

where

$$
\frac{d}{d t_{b}}=\frac{\partial}{\partial t}+\left(u-\frac{K_{v}}{N} \frac{\partial N}{\partial x}\right) \frac{\partial}{\partial x}+\left(v-\frac{K_{v}}{N} \frac{\partial N}{\partial z}\right) \frac{\partial}{\partial z}
$$

is the full derivative with respect to time for the bubble field. Here, $\rho_{g}$ is the gas density in a bubble, $D$ is the molecular diffusion coefficient, $p$ is the pressure in the fluid [given by formulae (A9) and (A12) in Appendix A], Nu is the Nusselt number, related to the Peclet number $(\mathrm{Pe})$ by $^{2}$

$$
\mathrm{Nu}=\frac{2}{\pi} \mathrm{Pe}^{1 / 3}, \quad \text { where } \quad \mathrm{Pe}=\frac{a v_{\infty}}{D},
$$

and $\kappa$ is the absorption coefficient, relating the pressure in the fluid to the volume concentration of the dissolved gas $C_{\infty}=\kappa p$.
Then, the pressure in the gas is found from the quasistatic relation (e.g., Ref. 14)

$$
p_{g}=p+\frac{2 \sigma}{a},
$$

where $\sigma$ is the surface tension coefficient. Finally, the gas density in a bubble is found from the equation

$$
\frac{d}{d t_{b}}\left(\frac{p_{g}}{\rho_{g}}\right)=0
$$

for temperature conservation in the ideal gas (see Ref. 17).

Thus, for a given internal wave field in the fluid, substituting expressions for $u(x, z, t), w(x, z, t)$, and $p(x, z, t)$ into Eqs. (1), (3), and (4), we can describe the dynamics of the bubble layer in the internal wave field. We impose constant boundary conditions for the radius and the gas density at the surface $z=0$

$$
a(x, 0, t)=a_{0}, \quad \rho_{g}(x, 0, t)=\rho_{g 0}
$$

and zero flux conditions at $z=-H$. Other boundary conditions, constants, and numerical schemes are discussed in the following sections.

\section{NUMERICAL SCHEME}

In the sequel, the governing Eqs. (1), (3), and (4) are solved by a first order implicit numerical scheme, which is based on the finite volume method. ${ }^{29} \Omega=\{(x, z): 0 \leq x \leq L$, $-H \leq z \leq 0\}$, with length $L$ equal to the internal wavelength $\lambda$ and depth $H$ taken to be twice the pycnocline depth $h$.

At a given time step $t_{n}$, we first obtain the gas density $\rho_{g}\left(x_{i}, z_{j}, t_{n}\right)$ from Eq. (4), then we get the bubble radius from Eq. (3), and finally we obtain $N\left(x_{i}, z_{j}, t_{n}\right)$, solving Eq. (1). Here we show the finite volume discretization of Eq. (1) [Eqs. (3) and (4) are written similarly]

$$
\begin{aligned}
& \frac{N_{i j}^{n+1}-N_{i j}^{n}}{\Delta t}+\frac{1}{\Delta x}\left(u_{i+1 / 2, j}^{n+1}-u_{i-1 / 2, j}^{n+1}\right) N_{i j}^{n+1}+\frac{1}{\Delta x}\left[\max \left(u_{i-1 / 2, j}^{n+1}, 0\right)-\min \left(u_{i+1 / 2, j}^{n+1}, 0\right)\right] N_{i j}^{n+1}+\frac{1}{\Delta x} \min \left(u_{i+1 / 2, j}^{n+1}, 0\right) N_{i+1, j}^{n+1} \\
& \quad-\frac{1}{\Delta x} \max \left(u_{i-1 / 2, j}^{n+1}, 0\right) N_{i-1, j}^{n+1}+\frac{1}{\Delta z}\left(v_{i, j+1 / 2}^{n+1}-v_{i, j-1 / 2}^{n+1}\right) N_{i j}^{n+1}+\frac{1}{\Delta z}\left[\max \left(v_{i, j-1 / 2}^{n+1}, 0\right)-\min \left(v_{i, j+1 / 2}^{n+1}, 0\right)\right] N_{i j}^{n+1} \\
& \quad+\frac{1}{\Delta z} \min \left(v_{i, j+1 / 2}^{n+1}, 0\right) N_{i, j+1}^{n+1}-\frac{1}{\Delta z} \max \left(v_{i, j-1 / 2}^{n+1}, 0\right) N_{i, j-1}^{n+1} \\
& =\frac{1}{\Delta x}\left(K_{v, i+1 / 2, j} \frac{N_{i+1, j}^{n+1}-N_{i j}^{n+1}}{\Delta x}-K_{v, i-1 / 2, j} \frac{N_{i j}^{n+1}-N_{i-1, j}^{n+1}}{\Delta x}\right) \\
& \quad+\frac{1}{\Delta z}\left(K_{v, i, j+1 / 2} \frac{N_{i, j+1}^{n+1}-N_{i j}^{n+1}}{\Delta z}-K_{v, i, j-1 / 2} \frac{N_{i j}^{n+1}-N_{i, j-1}^{n+1}}{\Delta z}\right)-\sigma_{*} N_{i j}^{n+1} .
\end{aligned}
$$

The numerical solution is obtained by an iterative procedure until convergence. We imposed periodic boundary conditions at $x=0$ and $x=L$

$$
N(0, z, t)=N(L, z, t), \quad a(0, z, t)=a(L, z, t), \quad \rho_{g}(0, z, t)=\rho_{g}(L, z, t) .
$$




\section{STRONG WIND: INTERNAL WAVE MODIFICATION OF THE BUBBLE LAYER}

According to experimental observations (see Ref. 1), surface waves start to break at wind speeds of about $2-3 \mathrm{~m} / \mathrm{s}$, and in winds of about $7 \mathrm{~m} / \mathrm{s}$, the bubbles form a continuous layer just below the surface. Here we consider the formation of the bubble layer at high wind speeds, when the source at the surface can be treated as homogeneous and, as discussed above, we use a simple model $q(x, z, t)=q_{0} \delta(z+\epsilon)$ where $q_{0}$ is a constant.

To model structure formation in the bubble layer, we choose the following parameters of the computational domain: $L=\lambda=200 \mathrm{~m}, h=10 \mathrm{~m}$, and $H=20 \mathrm{~m}$. Following Ref. 2, we take $\mu_{l}=10^{-3} \mathrm{~Pa} \mathrm{~s}, \quad \sigma=0.036 \mathrm{~N} / \mathrm{m}$, $D=2 \times 10^{-9} \mathrm{~m}^{2} / \mathrm{s}$, and $\kappa=2.1 \times 10^{-7} \mathrm{~kg} /\left(\mathrm{m}^{3} \mathrm{~Pa}\right)$, while $\rho_{1}=999 \mathrm{~kg} / \mathrm{m}^{3}$ and $\rho_{2}=1000 \mathrm{~kg} / \mathrm{m}^{3}$. We assume that at the surface, $a_{0}=50 \mu \mathrm{m}$ and $\rho_{g 0}=1.2\left(1+2 \sigma / p_{a} a_{0}\right) \mathrm{kg} / \mathrm{m}^{3}$, where $p_{a}=10^{5} \mathrm{~Pa}$ is the atmospheric pressure. The coefficient of turbulent diffusion $K_{v}$ is taken in the form ${ }^{7}$

$$
K_{v}=K_{v 0}-c_{k} \sqrt{\frac{\rho_{g 0} c_{d}}{\rho_{l}}} W z
$$

where $c_{k}=0.4$ is the Von Karman constant, $c_{d}=1.3 \times 10^{-3}$ is the drag coefficient, $\rho_{g 0}$ is the gas density at the surface, $W$ is the wind speed, and $K_{v 0}$ is the turbulent diffusion coefficient at the interface

$$
K_{v 0}=-c_{k} \sqrt{\frac{\rho_{g} c_{d}}{\rho_{l}}} W z_{s} .
$$

In our calculations, we use $z_{s}=-1.5 \mathrm{~m}$, so formula (6) gives $K_{v}=5.2 \times 10^{-4}(1.5-z) W$.

Following Ref. 30, we consider $q_{0}$ in the form $q_{0}=\hat{q}_{0}(W / 10)^{\eta}$, where $\hat{q}_{0}$ is the source term value for $\hat{W}=10 \mathrm{~m} / \mathrm{s}$ and $\eta$ is an unknown parameter. The source term $\hat{q}_{0}$ and the dissolution rate $\sigma_{*}$ in Eq. (1) can be estimated as follows. Let us suppose that for $W=\hat{W}$, the void concentration changes exponentially with depth according to the empirical law ${ }^{2}$

$$
\hat{\alpha}_{g}=\hat{\alpha}_{g 0} \exp (z / 0.7),
$$

where $\hat{\alpha}_{g 0}=10^{-5}$, which is in good agreement with experimental data (see Refs. 7 and 10, for instance). From Eqs. (1) and (2), the depth-dependent number distribution $\hat{N}=\hat{N}(z)$ of bubbles is described by the following problem:

$$
\begin{aligned}
& \frac{d}{d z}\left\{\hat{K}_{v} \frac{d \hat{N}}{d z}-v_{\infty} \hat{N}\right\}-\sigma_{*} \hat{N}=0, \\
& K_{v 0} \frac{d \hat{N}}{d z}-\left.v_{\infty} \hat{N}\right|_{z=0}=\hat{q}_{0},\left.\quad \frac{d \hat{N}}{d z}\right|_{z \rightarrow-\infty}=0,
\end{aligned}
$$

where we simplified the problem by assuming infinite depth, which is clearly a good approximation. Following the analysis of Buckingham, ${ }^{7}$ the solution to this problem is

$$
\begin{aligned}
& \hat{N}(z)=\hat{N}(0) F(z), \quad \hat{N}(0)\left[K_{v 0} \frac{d F}{d z}\right. \\
& \text { where } \quad F(z)=\frac{\xi(z)^{-\nu} K_{\nu}[\xi(z)]}{\xi(0)^{-\nu} K_{\nu}[\xi(0)]}, \\
& \xi(z)=\frac{2 \sigma_{*}^{1 / 2}\left(K_{v 0}-\alpha z\right)^{1 / 2}}{\alpha}, \\
& \alpha=c_{k} \sqrt{\frac{\rho_{g_{0} c_{d}}}{\rho_{l}}} \hat{W}, \quad \nu=\frac{v_{\infty}}{\alpha} .
\end{aligned}
$$

Here $K_{\nu}(\cdot)$ is the modified Bessel function of the second kind. This then determines $\hat{q}_{0}$ in terms of $\hat{N}(0)$. Note that in the limit $\nu \rightarrow \infty$ with $z$ fixed, that is we let $\alpha \rightarrow 0$, the solution (8) reduces to

$$
\begin{aligned}
& F(z)=\exp \left(\frac{v_{\infty}+\sqrt{v_{\infty}^{2}+4 K_{v 0} \sigma_{*}}}{2 K_{v 0}} z\right), \\
& \hat{N}(0)=\frac{2 \hat{q}_{0}}{\left(\sqrt{v_{\infty}^{2}+4 K_{v 0} \sigma_{*}}-v_{\infty}\right)} .
\end{aligned}
$$

When applicable, this expression is much easier to use in practice than Eq. (8). For instance, assuming that all bubbles close to the surface have an approximately constant radius $a=a_{0}=$ const and the given void fraction $\hat{\alpha}_{g 0}=\frac{4}{3} \pi \hat{N}(0) a_{0}^{3}$, which is proportional to $\hat{N}(0)$, formulae (7) and (9) allow some simple estimates of $\hat{q}_{0}$ and $\sigma_{*}$.

Next to find $\eta$ we use the empirical formula ${ }^{31}$

$$
d=0.4(W-2.5) \text {, }
$$

where $d$ (meters) is an average penetration depth of the bubble clouds (may be treated as the depth at which void concentration equals to void concentration for $W=2.5 \mathrm{~m} / \mathrm{s}$ at the surface). Solving Eq. (1) as above for an undisturbed fluid for different values of $W$ we found that the best approximation of Eq. (10) is achieved for $\eta \approx 3$, which is the same as obtained in Ref. 30.

Figure 2 shows the distributions of the acoustic speed, number density of bubbles, their radii, and void fraction for a pure two-layer fluid periodic internal wave of amplitude $3 \mathrm{~m}$ and for a wind speed $10 \mathrm{~m} / \mathrm{s}$. The internal wave propagates from left to the right. The speed of sound in the fluid with gas bubbles is calculated according to the following formula (e.g., Ref. 14):

$$
c^{2}=\frac{\rho_{g} \rho_{l} c_{g}^{2} c_{l}^{2}}{\left[\alpha_{g} \rho_{g}+\left(1-\alpha_{g}\right) \rho_{l}\right]\left[\alpha_{g} \rho_{l} c_{l}^{2}+\left(1-\alpha_{g}\right) \rho_{g} c_{g}^{2}\right]},
$$

where $c_{g}=290 \mathrm{~m} / \mathrm{s}$ and $c_{l}=1500 \mathrm{~m} / \mathrm{s}$ are the values of sound speed in a gas and a fluid. The numerical calculations were carried out on a homogeneous grid with 40 cells in the horizontal and 80 cells in the vertical direction. The initial conditions correspond to undisturbed fluid. The graphs are plotted for the time $t=10 T$, where $T$ is the period of the internal wave, for which the numerical solution becomes strictly periodic. 
a)

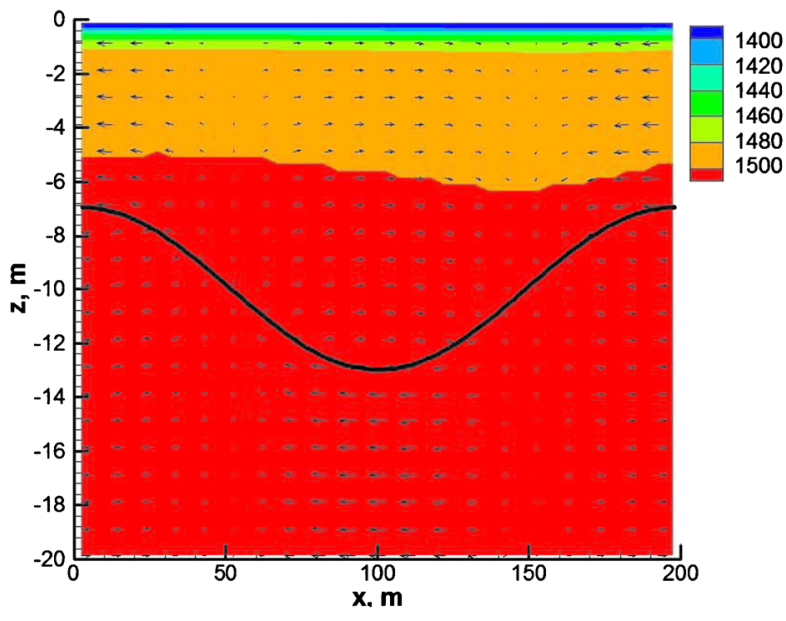

c)

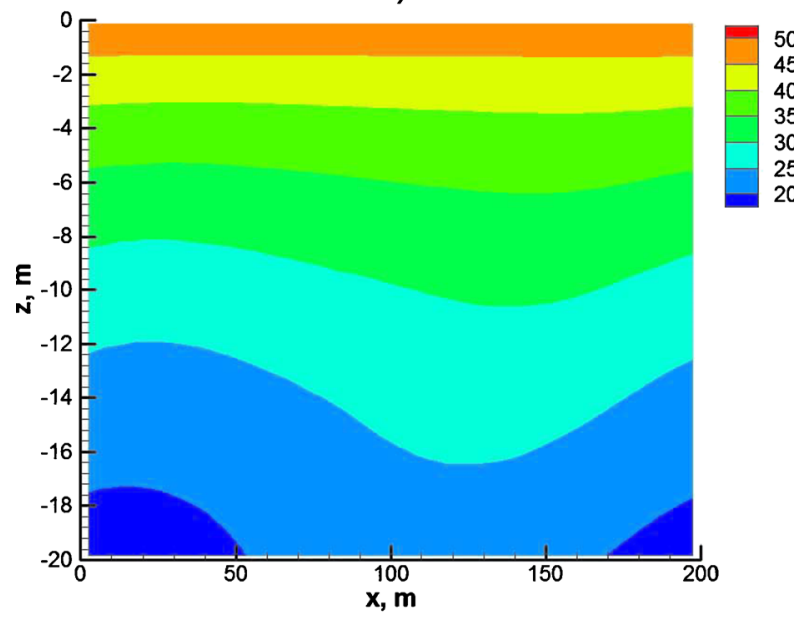

b)

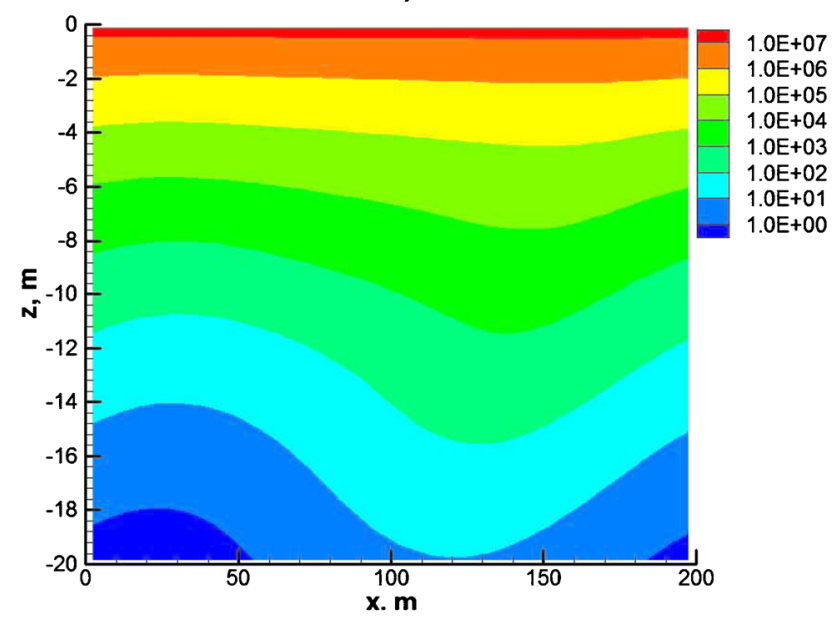

d)

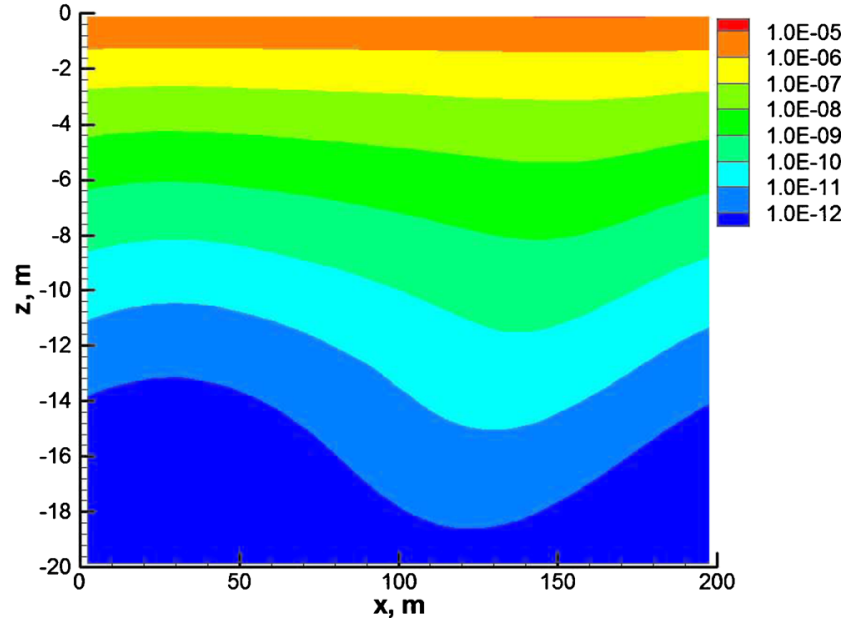

FIG. 2. (Color online) (a) Acoustic speed (meters per second), (b) number density of bubbles $\left(1 / \mathrm{m}^{3}\right)$, (c) radius (micrometers), and (d) void fraction ( $\alpha$ ) for a two-layer periodic internal wave of $3 \mathrm{~m}$ amplitude [shown in (a)] and for a wind speed $W=10 \mathrm{~m} / \mathrm{s}\left(\hat{q}_{0} \approx 1.38 \times 10^{3} \mathrm{~m}^{-3} \mathrm{~s}^{-1}\right.$ and $\left.\sigma_{*} \approx 3.10 \times 10^{-2} \mathrm{~s}^{-1}\right)$.

Our results show that the periodic internal wave produces a displacement in the surrounding fluid with orbital velocities up to $5-10 \mathrm{~cm} / \mathrm{s}$. Some bubbles move toward the interface and others are carried down by the flow. Consequently, we observe horizontally inhomogeneous profiles of the bubble parameters not only in the neighborhood of the pycnocline, but also away from it. For a fixed depth the void fraction of bubbles shows a considerable horizontal variability, being at maximum in the downwelling area behind the crest of the internal wave. It is necessary to note, however, that in this current study we have neglected the effects of Langmuir circulation, which can also contribute significantly to the organization of bubbles (e.g., Refs. 32-34). Nevertheless, we believe the mechanism described in this paper to be dominant at least in the cases when the average distance between windrows is much smaller than the wavelength of the internal wave or when the internal wave has a large amplitude.

Figure 3 shows the distributions of acoustic speed and void fraction for the wind speeds $W=7.5 \mathrm{~m} / \mathrm{s}$ and $W=12.5 \mathrm{~m} / \mathrm{s}$. Since the turbulent diffusion coefficient and the value of gas volume fraction at the surface depend on the wind speed, the penetration of bubbles increases and the horizontal inhomogeneities become more evident with the increase of $W$.

Figure 4 shows the $\log \left(M_{v}\right)$ distribution for $W$ $=7.5 \mathrm{~m} / \mathrm{s}$ and $W=12.5 \mathrm{~m} / \mathrm{s}$. Here $M_{v}$ is the acoustic scattering cross section per unit volume (e.g., Ref. 2)

$$
M_{v}=N \sigma_{v}, \quad \sigma_{v}=\frac{4 \pi a^{2}}{\left[\left(\omega_{0} / \omega\right)^{2}-1\right]^{2}+\Psi^{2}},
$$

where

$$
\Psi=\frac{3 \rho_{g} c_{g}^{2}}{a \rho_{l} c_{l} \omega}, \quad \omega_{0}=\frac{\sqrt{3} c_{g}}{2 \pi a},
$$

and $\omega=600 \mathrm{kHz}$ is the typical sonar frequency. Similar to the other parameters, the horizontal distribution of $\log \left(M_{v}\right)$ in the subsurface layer approximately copies the pycnocline wave with a shift to the right (in the direction of the internal wave propagation). 
a)
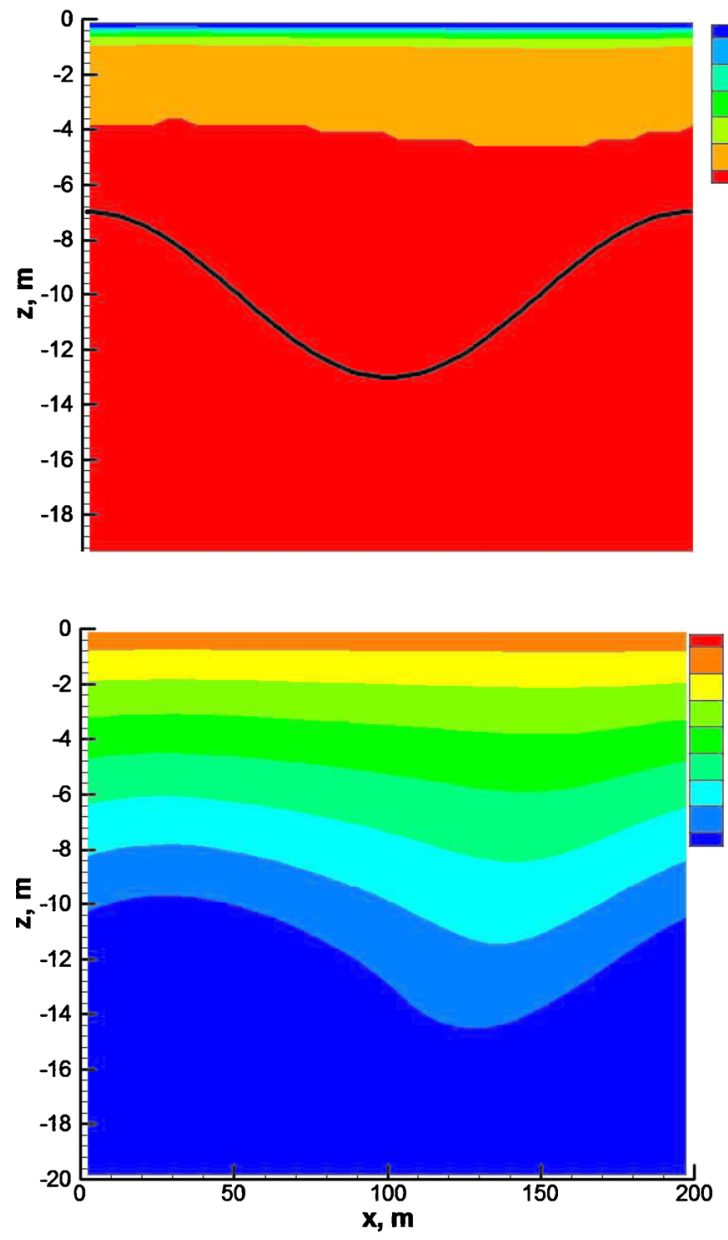

b)
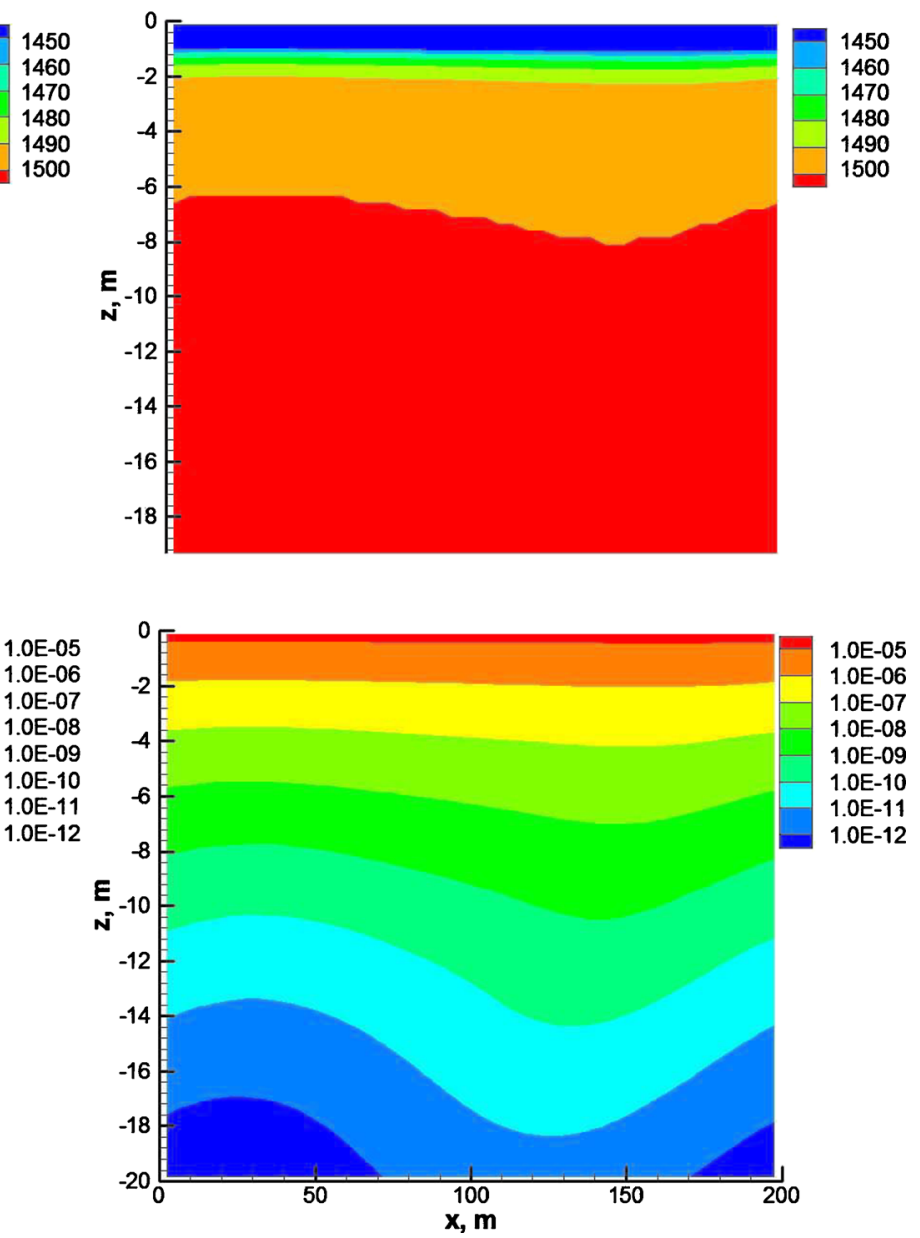

FIG. 3. (Color online) (Top row) Acoustic speed (meters per second) and (bottom row) void fraction ( $\alpha$ ) for a two-layer periodic internal wave of 3 m amplitude [shown in (a)] for a wind speed (a) $W=7.5 \mathrm{~m} / \mathrm{s}$ and (b) $W=12.5 \mathrm{~m} / \mathrm{s}$.

$$
W=7.5 \mathrm{~m} / \mathrm{s}
$$

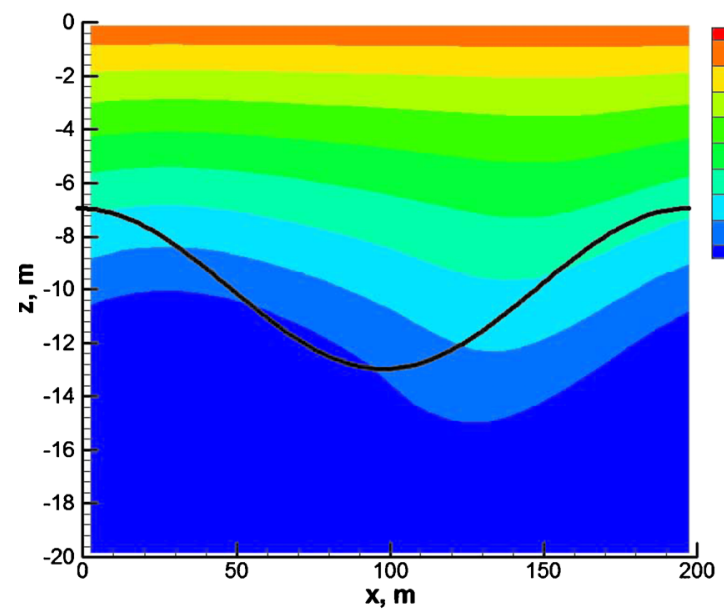

$\mathrm{W}=12.5 \mathrm{~m} / \mathrm{s}$

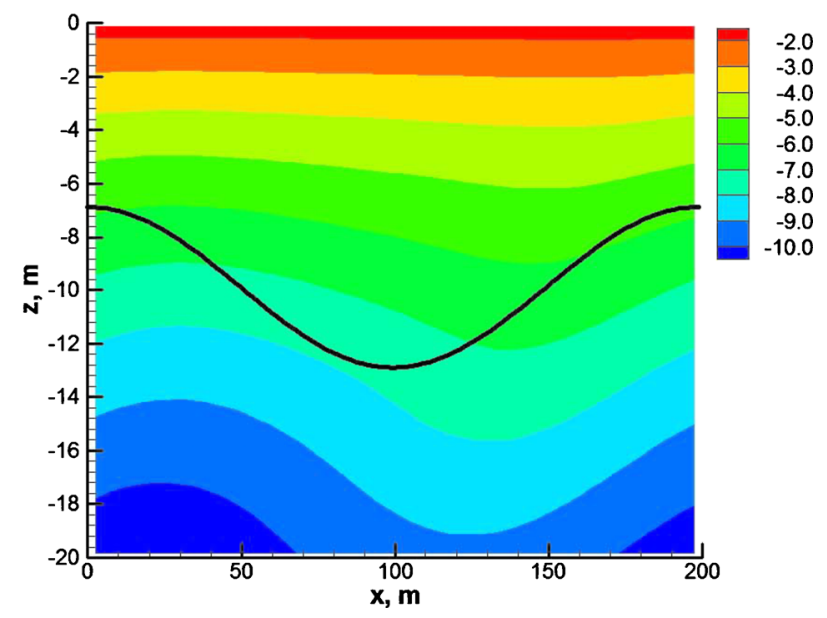

FIG. 4. (Color online) Logarithm of the scattering intensity $M_{v}$ (measured in units $\mathrm{m}^{-1}$ ) for a two-layer fluid periodic internal wave of 3 m amplitude for two values of a wind speed $W=7.5 \mathrm{~m} / \mathrm{s}$ and $W=12.5 \mathrm{~m} / \mathrm{s}$. 

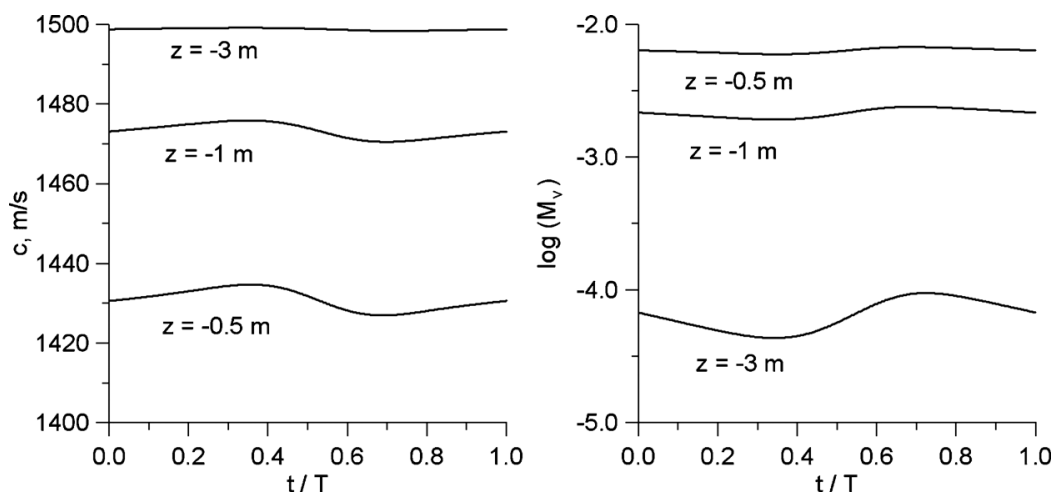

FIG. 5. Variations of sound speed $c$ and logarithm of the scattering intensity $M_{v}$ (measured in units $\mathrm{m}^{-1}$ ) with time for $W=10 \mathrm{~m} / \mathrm{s}$ for three different depths: $z=-0.5 \mathrm{~m}, z=-1 \mathrm{~m}$, and $z=-3 \mathrm{~m}$.

In Fig. 5, the time variations of the acoustic speed $c$ and scattering intensity $\log \left(M_{v}\right)$ are displayed for $W=10 \mathrm{~m} / \mathrm{s}$ and three different depths: $z=-0.5 \mathrm{~m}, z=-1 \mathrm{~m}$, and $z=-3 \mathrm{~m}$.

The character of the distribution of bubbles in the subsurface layer depends on the parameters of the internal wave (wavelength, amplitude, and densities of the fluid layers) and on the average bubble radius. Numerical simulations with different parameters of the internal wave showed that the heterogeneity of the bubble layer parameters (number of bubbles, void fraction, and speed of sound) becomes more evident as the orbital velocities of fluid particles increase. Nevertheless, the solution is qualitatively the same as in Figs. 2-5.

\section{INTERNAL WAVE AS THE MAIN CAUSE OF SURFACE WAVE BREAKING}

In this section, we consider the case when the breaking of the surface waves is primarily caused by their modulation with a horizontal current, due to an internal wave propagating along a shallow pycnocline. This situation is typical for weak winds when the wind speed is less than $5 \mathrm{~m} / \mathrm{s}$. We assume that the internal and surface waves are copropagating. In this case, we need to find the spatially inhomogeneous source term for the injected bubbles $q(x, z, t)$ by relating the surface wave breaking to the internal wave field. Here we adapt an approach used in a study of the role of tidally forced flow-topography interaction in the aeration of the subsurface waters. ${ }^{21}$

We are concerned with surface waves riding on a long internal wave $u_{i}=u_{i}\left(x-c_{i} t, z\right)$, slowly varying relative to the surface wave. There exists an extensive literature on the modulation of surface waves by a background current, beginning with Refs. 35-38. These theories can be adapted to the modulation of surface waves by an internal wave, where it is assumed that the background current is that determined by the horizontal velocity field at the free surface due to the underlying internal wave (see, for instance, Refs. 39 and 40). An alternative model commonly used for the interpretation of images of the surface signature of internal waves was introduced in Ref. 41; here the surface wave field is maintained by a strong wind and the internal wave is regarded as a perturbation to this field. A summary of these studies is given in Appendix B.
Here we assume the absence of the strong wind. Then, in the reference frame which moves with the speed of the internal wave, the internal wave current becomes $V(X)=u_{i}(X, 0)-c_{i}$, where $X=x-c_{i} t$ and the amplitude of the modulated deep-water surface wave is given by

$$
\begin{aligned}
\frac{a_{s}^{2}(X)}{a_{s 0}^{2}}= & \frac{1}{2}\left[\frac{V(X)}{c_{0}}+\frac{1}{4}\right]^{-1 / 2} \\
& \times\left\{\frac{1}{2}+\left[\frac{V(X)}{c_{0}}+\frac{1}{4}\right]^{1 / 2}\right\}^{-2}, \quad \text { where } c_{0}=\left(g / k_{0}\right)^{1 / 2},
\end{aligned}
$$

while the wave number is given by

$$
\frac{\kappa}{\kappa_{0}}=\frac{c_{0}^{2}}{c^{* 2}}, \quad \text { where } \quad c^{*}=\frac{c_{0}}{2}+c_{0}\left[\frac{V(X)}{c_{0}}+\frac{1}{4}\right]^{1 / 2}
$$

(for details see Appendix B). For $V$ positive and increasing, the surface wave energy $a_{s}^{2}$ decreases, while for $V$ negative and decreasing, $a_{s}^{2}$ increases to infinity at the stopping velocity $-c_{0} / 4$. In this case, wave breaking will occur before the stopping velocity. Note that we can use the formulae in Appendix A to relate $V(X)=u_{i}(X)-c_{i}$ to the internal wave amplitude at the pycnocline. In particular, the surface current is opposite in sign to the phase speed over the wave crest and in sympathy over the wave trough, with the maximum downwelling region in between. Thus the surface waves steepen as they approach the internal wave crest, and diminish as they approach the wave trough.

Expression (11) predicts that when the surface waves propagate into an opposing current of increasing strength $V<0, V_{X}<0$, then the surface wave amplitude grows indefinitely up to the stopping velocity. In practice, the surface waves will break at locations $X_{c}$ determined by the criterion

$$
\kappa a_{s}=\left(\kappa a_{s}\right)_{c}=S_{c},
$$

where $S_{c}$ is the critical steepness, after which the waves break. In Ref. 21, it was suggested that $S_{c}$ can vary in practice over the range $0.15-0.5$ and we note that the theoretical critical value for the wave of maximum steepness is $S_{c}=0.44$ (see Ref. 42, for instance). Combining the criterion (13) with Eqs. (11) and (12), we get that 


$$
\begin{aligned}
\frac{\kappa^{2} a_{s}^{2}}{\kappa_{0}^{2} a_{s 0}^{2}}= & \frac{1}{2}\left[\frac{V(X)}{c_{0}}+\frac{1}{4}\right]^{-1 / 2} \\
& \times\left\{\frac{1}{2}+\left[\frac{V(X)}{c_{0}}+\frac{1}{4}\right]^{1 / 2}\right\}^{-6}, \quad \frac{\left(\kappa a_{s}\right)_{c}}{\kappa_{0} a_{s 0}}=\frac{S_{c}}{\kappa_{0} a_{s 0}},
\end{aligned}
$$

where we recall that the subscript 0 indicates values at the location where $V=0$. It is readily shown from Eq. (14) that $\kappa a_{s}$ increases as $V(X)<0$ decreases. This expression is now applied to the internal wave with $V(X)=u_{i}(X, 0)-c_{i}$, where the internal wave horizontal velocity field $u_{i}$ is found from expression (A7) in the upper layer and whose interfacial displacement field is given by Eq. (A6). These expressions then define a possible breaking zone which lies over the crest of the internal wave, since this is where $V(X)$ reaches its minimum negative value. Let $X_{M}=2 \pi / k$ denote the location of one internal wave crest, where $k$ is the internal wave number. Then the possible breaking zone is defined by $X_{c 1}<X<X_{c 2}$, where $X_{c 1}<X_{M}$ and since the internal wave (A6) is symmetric about the crest, $X_{c 2}=2 X_{M}-X_{c 1}$. For a periodic internal wave, this breaking zone is then repeated periodically, around each internal wave crest.

In this zone, we need to determine the energy released by wave breaking and available for the injection of bubbles. The wave energy at any location $X$ in the absence of breaking is $E=\rho_{l} g a_{s}^{2} / 2$, where $a_{s}(X)$ is given by Eq. (11). Next we follow Ref. 21 and assume that the wave energy of a breaking wave is $\rho_{l} g \widetilde{a}_{s}^{2} / 2$, where the breaking wave amplitude is defined by the condition that $\widetilde{a}_{s} \kappa=S_{c}$ throughout the breaking zone and the wave number $\kappa$ is defined by Eq. (12), the same value as in the absence of breaking. Hence the energy available to inject bubbles is the difference between these, given by

$$
\widetilde{E}_{1}(X)=E_{0}\left\{\frac{a_{s}^{2}(X)}{a_{s 0}^{2}}-\frac{S_{c}^{2}}{a_{s 0}^{2} \kappa_{0}^{2}} \frac{\kappa_{0}^{2}}{\kappa^{2}(X)}\right\}, \quad E_{0}=\frac{1}{2} \rho_{l} g a_{s 0}^{2} .
$$

Here $X$ is constrained to lie in the breaking zone. We then further assume that a fraction $E_{b r}=\varepsilon \widetilde{E}_{1}$ of this breaking wave energy is available for the injection of bubbles and, following Ref. 21 , we set $\varepsilon=0.003$, appropriate for spilling breakers.

On the other hand, the energy per unit volume needed to submerge a single bubble of radius $a$ to a depth $z$ is given by

$$
E_{b}=-\frac{4}{3} \pi a^{3} \rho_{l} g z
$$

Then, from an energy balance, we get that

$$
E_{b r}=\varepsilon \widetilde{E}_{1}=-\int_{-H}^{0} \frac{4}{3} \pi a^{3} \rho_{l} g z \tilde{N} d z
$$

where $\tilde{N}$ is the number of newly born bubbles per unit surface area, given by

$$
\tilde{N}=\beta_{0}(X) \exp \left(\frac{z}{\gamma}\right),
$$

with $\gamma=a_{s}(X) / 4$ (see Ref. 21), where $\beta_{0}(X)$ can be found from Eq. (16). If we make a simplifying assumption that all newly born bubbles have the same initial radius $a_{0}=50 \mu \mathrm{m}$ (equal to the bubble radius at $z=0$, when the fluid is in rest), then we can integrate Eq. (16) explicitly, assuming that $H / \gamma \gg 1$,

$$
E_{b r}=\frac{4}{3} \pi \rho_{l} g \beta_{0}(X) \gamma^{2} a_{0}^{3},
$$

and thus

$$
\tilde{N}(X, z)=\frac{3}{4 \pi} \frac{\varepsilon \widetilde{E}_{1}(X)}{\rho_{l} g \gamma^{2} a_{0}^{3}} \exp \left(\frac{z}{\gamma}\right)
$$

is the number density of newly injected bubbles over the breaking zone. These bubbles are injected over every period $P_{s}=2 \pi / \Omega_{0}$ of the surface wave and so finally we get an upper estimate for the source term in the possible breaking zone,

$$
q_{\max }(X, z)=\frac{\tilde{N}(X, z)}{P_{s}},
$$

while it is zero outside this zone.

Expression (18) assumes that the breaking surface waves can be represented as a steady-state field over the breaking zone. In that representation, the entire surface wave field (B4) is replaced by breaking waves. However, assuming that the breaking is due to spilling breakers, in practice only a small region around each surface wave crest will create breaking wave energy available for the creation of bubbles. In this scenario, at each particular fixed location $X$ in the breaking zone, we use a local representation of the surface wave defined by Eq. (B4), namely,

$$
\zeta_{s} \approx a_{s}(X) \cos \left[\kappa(X) X-\Omega_{0}\left[T-T_{0}\right]\right],
$$

where $T_{0}$ is a phase constant. Recall that in this breaking zone $\kappa(X) a_{s}(X) \geq S_{c}$ and so, for each fixed $X$, the local steepness $\kappa(X) \zeta_{s} \geq S_{c}$ over some small time interval $T_{1}<T<T_{2}$, where surface wave breaking occurs. Without loss of generality, we can suppose that $T-T_{0} \geq\left\{\kappa(X) a_{s}(X)-3 \pi / 2\right\} / \Omega_{0}$. Then let $T_{1}$ denote that moment of time when the local breaking condition

$$
\kappa(X) \zeta_{s}(X, T)=\kappa(X) a_{s}(X) \cos \left[\kappa(X) X-\Omega_{0}\left[T-T_{0}\right]\right] \geq S_{c}
$$

is first satisfied at this fixed point $X$, that is, the equality that holds in Eq. (20). Then as $T$ increases, we let $T_{M}$ denote that moment of time when $\zeta_{s}(X, T)$ reaches its maximum value for the first time. Although technically the breaking condition (20) continues to hold until $T_{2}=2 T_{M}-T_{1}>T_{M}$; we note that for $T_{M}<T<2 T_{M}-T_{1}$, the rate of energy loss is negative and no bubbles will be injected. Then $\Delta T_{b}=T_{M}-T_{1}$ is the duration of a breaking event at $X$, which is then repeated periodically with a period $P_{s}=2 \pi / \Omega_{0}$.

The next task is to find the energy $\widetilde{E}_{2}$ generated by this breaking wave which is available for the injection of 
bubbles. To this end, we assume that over the time interval $\Delta T_{b}$, the crest of the local expression (19) for a nonbreaking wave is replaced by a "flat cap" of constant amplitude $\widetilde{a}_{s}$, determined by the breaking condition that $\kappa \widetilde{a}_{s}=S_{c}$ [compare the analogous discussion above leading expression (15)]. Then the wave potential energy lost in this breaking zone is available for the injection of bubbles. In the absence of breaking, the potential energy is $\rho_{l} g \zeta_{s}^{2} / 2$ where $\zeta_{s}$ is given by Eq. (19) and is time-dependent. As above, the potential energy of the breaking wave is $\rho_{l} g a_{s}^{2} / 2$. Hence the available energy is

$$
\begin{aligned}
\widetilde{E}_{2}(X, T)= & E_{0}\left\{\frac{a_{s}^{2}(X)}{a_{s 0}^{2}} \cos ^{2}\left[\kappa(X) X-\Omega_{0}\left[T-T_{0}\right]\right]\right. \\
& \left.-\frac{S_{c}^{2}}{a_{s 0}^{2} \kappa_{0}^{2}} \frac{\kappa_{0}^{2}}{\kappa^{2}(X)}\right\} .
\end{aligned}
$$

This expression holds only in the possible breaking zone over the time interval $\Delta T_{b}$, where the inequality (20) holds; $E_{0}$ is defined above in Eq. (15), while the terms $a_{s}^{2}(X) / a_{s 0}^{2}$ and $\kappa_{0}^{2} / \kappa^{2}(X)$ are defined by Eqs. (B11) and (B9), respectively. The remaining calculation now proceeds as above for the steady-state case, where we replace $\widetilde{E}_{1}$ with $\widetilde{E}_{2}$ in each of the expressions leading to Eq. (17). The essential difference is that now the rate of injection of bubbles occurs over the interval $\Delta T_{b}$ and is repeated over many surface wave periods $P_{s}=2 \pi / \Omega_{0}$. Then we define the time-dependent upper estimate for the source term by

$$
\begin{aligned}
& q_{\max }(X, z, T)=\frac{\partial \tilde{N}(X, z, T)}{\partial T} \\
& \text { for } T_{1}+k P_{s}<T<T_{M}+k P_{s}, \quad k=0,1,2, \cdots, \\
& \text { or } q_{\max }(X, z, T)=0 \quad \text { otherwise, } \\
& \text { where } \tilde{N}(X, z, T)=\frac{3}{4 \pi} \frac{\varepsilon \widetilde{E}_{2}(X, T)}{\rho_{l} g \gamma^{2} a_{0}^{3}} \exp \left(\frac{z}{\gamma}\right) .
\end{aligned}
$$

As before, this expression holds only when $X$ lies in the spatial possible breaking zone defined by Eq. (14) (repeated near every internal wave crest). Note that indeed $q(X, z, T)$ $\geq 0$ as required for bubble injection. It can be shown that averaging expression (21) over the phase $T_{0}$ recovers the steady formula (18).

For our numerical results, we show only those using the time-dependent source term (21). Averaged results are similar to those obtained using the steady-state form (18). To account for the attenuation of the breaking surface wave, as well as for possible deviation toward predictions of the theory in Ref. 41 (see discussion at the end of Appendix B), we use a simple phenomenological model, where we replace the upper estimate obtained above by the expression

$$
q(X, z, T)=q_{\max }(X, z, T) e^{-\left(X-X_{c 1}\right) / \delta},
$$

for $X_{c 1} \leq X \leq X_{c 2}$ and similarly for other possible breaking zones. Here, the choice $1 / \delta \rightarrow 0$ recovers the upper estimate for $q$. We then can choose the value of this constant $\delta$, which represents the effective width of the breaking zone. In the following, we compare the results obtained for the upper estimate (21) and for the attenuated expression (22), where the constant $\delta=X_{M}-X_{c 1}$, i.e., breaking effectively takes place in the area $X_{c 1}<X<X_{M}$. (We assume that the value of this constant can be potentially obtained more accurately from experimental and observational results.)

Figure 6 shows the distribution of void fraction for the wind speed $W=2.5 \mathrm{~m} / \mathrm{s}$, when bubbles are injected by breaking surface waves due to interaction with a copropagating internal wave. We compare the distributions obtained for the source term given by the expressions (21) [shown in Fig. 6(a)] and (22) [shown in Fig. 6(b)]. Figure 6 also shows the averaged value of the source term $\bar{q}$ for these two expressions, where

$$
\bar{q}(X)=\frac{1}{5 P_{s}} \int_{5 P_{s}}^{10 P_{s}} \int_{-H}^{0} q(X, z, T) d z d T .
$$

Figure 7 shows the acoustic speed corresponding to the distribution in Fig. 6(b) [also reproduced in Fig. 7(b)]. To model the surface waves we used the following parameters: $S_{c}=1 / 2, a_{s 0}=0.1 \mathrm{~m}, \lambda_{0}=2 \pi / \kappa_{0}=2.5 \mathrm{~m}\left(\kappa_{0} a_{s 0}=0.25<S_{c}\right)$, and $\gamma=0.25 a_{s 0}$. According to our results, the region of injection of bubbles is again situated in the downwelling area behind the crest of the internal wave, as we found for the homogeneous input of bubbles from the surface for strong winds (see Fig. 2 for comparison). But in this case the inhomogeneity of the distribution of bubbles in the subsurface layer is much stronger, since bubbles are injected only through parts of the surface.

Figure 8 shows the averaged distribution of void fraction $\bar{\alpha}$ with depth for three different models of injection of bubbles, a homogeneous case $(W=10 \mathrm{~m} / \mathrm{s})$, and two inhomogeneous cases $[W=2.5 \mathrm{~m} / \mathrm{s}$, and we use either Eq. (21) or Eq. (22) for the source]. Here

$$
\bar{\alpha}(z)=\frac{1}{5 \lambda P_{s}} \int_{5 P_{s}}^{10 P_{s}} \int_{0}^{\lambda} \alpha(x, z) d x d T .
$$

We note that the averaged values of $\bar{q}$ and $\bar{\alpha}$ are close to the respective solutions corresponding to the steady source (18). The inhomogeneous input of bubbles leads to the more rapid decrease of the void fraction with depth, despite larger average value at the surface.

The detailed character of the bubble distribution in the subsurface layer depends on the parameters governing the breaking surface waves, that is, the initial wave number $\kappa_{0}$, the initial amplitude $a_{s 0}$, and the critical steepness $S_{c}$. The spatial distribution of $N$ for different values of wavelength and $S_{c}$ are shown in Figs. 9 and 10.

In Fig. 9, the number density of bubbles is shown for $a_{s 0}=0.1 \mathrm{~m}$ and three different values of $\lambda_{0}=2.6,2.5$, and $2.3 \mathrm{~m}\left(\kappa_{0} a_{s 0}=0.24,0.25\right.$ and 0.27 , respectively $)$. For 
a)
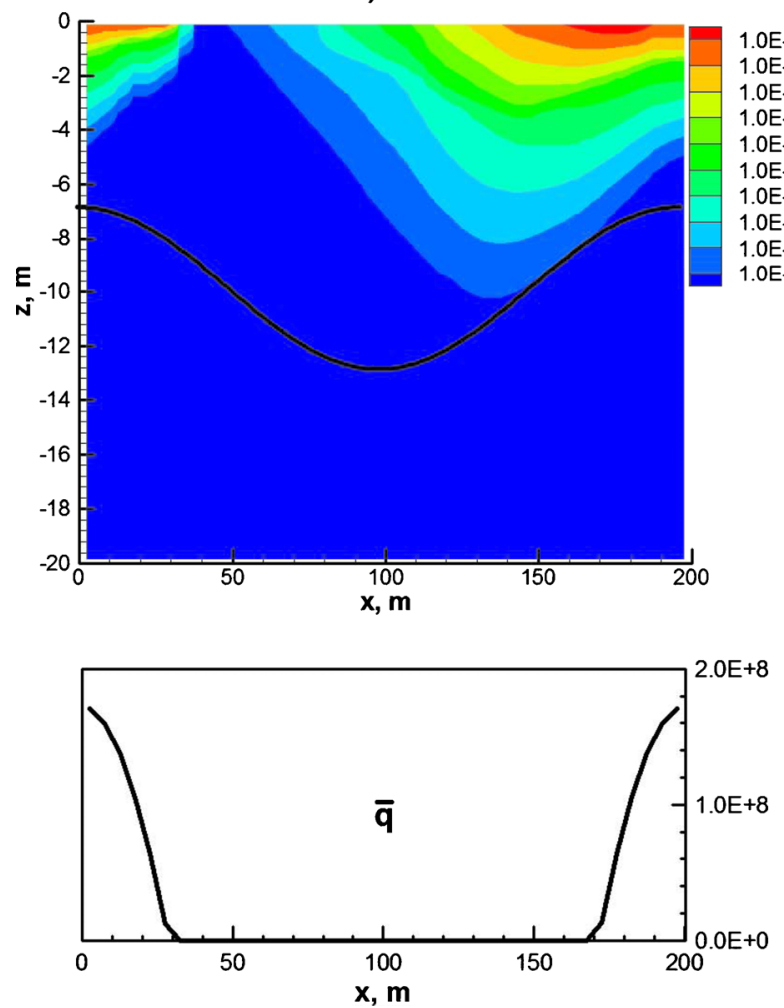

b)
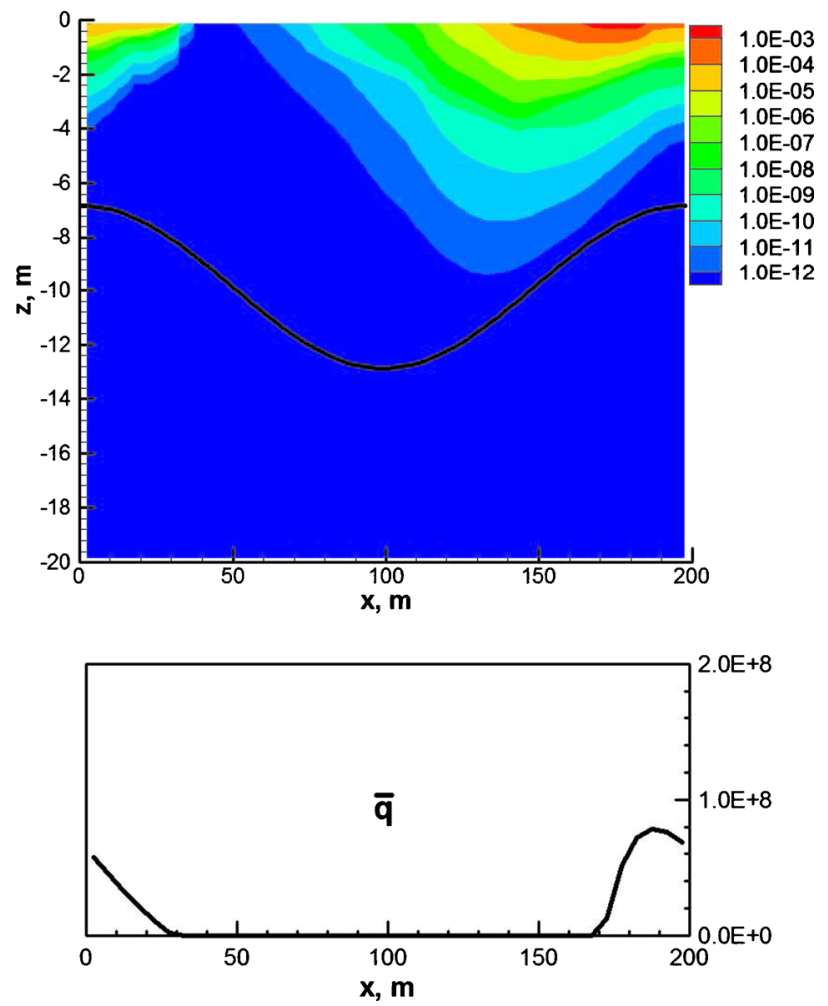

FIG. 6. (Color online) Void fraction of bubbles and averaged value of source term for periodic internal wave of 3 m amplitude and wind speed $W=2.5 \mathrm{~m} / \mathrm{s}$ (structure due to inhomogeneous breaking). The source term is calculated (a) using Eq. (21) and (b) using Eq. (22).

$\lambda_{0}=2.3 \mathrm{~m}$, surface waves begin to break at low horizontal velocities and the picture is closer to the case of homogeneous input of bubbles (e.g., Fig. 2) than in the remaining two cases. For $\lambda_{0}=2.6 \mathrm{~m}$, breaking of surface waves occurs in the narrow zones on the surface.

In Fig. 10, the same pictures are plotted for different $S_{c}$. We chose $S_{c}=0.5$ (basic value), 0.45 , and 0.4 (in this last case the initial steepness already exceeds the critical value). Here, $\lambda_{0}=2.5 \mathrm{~m}$ and $a_{s 0}=0.1 \mathrm{~m}$. As can be seen from the plots, the smaller is $S_{c}$ the wider is the region of surface waves breaking. Nevertheless, at a depth of $3 \mathrm{~m}$ and deeper, the character of the distribution of $N$ does not change noticeably because in this region, it is mainly defined by the slow convection.

The considerations above can be also applied to the case when the surface waves and the internal wave move in the opposite directions, with the appropriate changes for $V\left(X_{0}\right)$ and $\Omega_{0}$. However, $V\left(X_{0}\right)$ will always be positive for the case a)

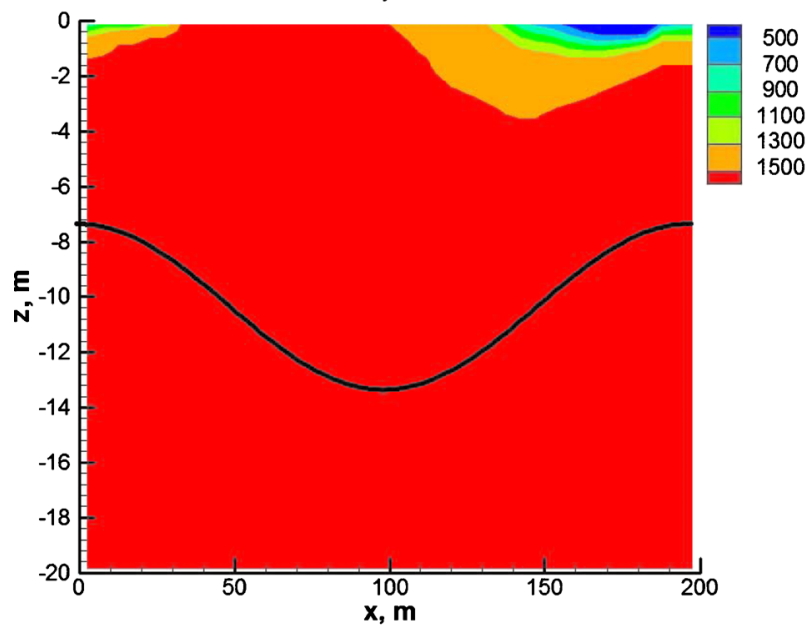

b)

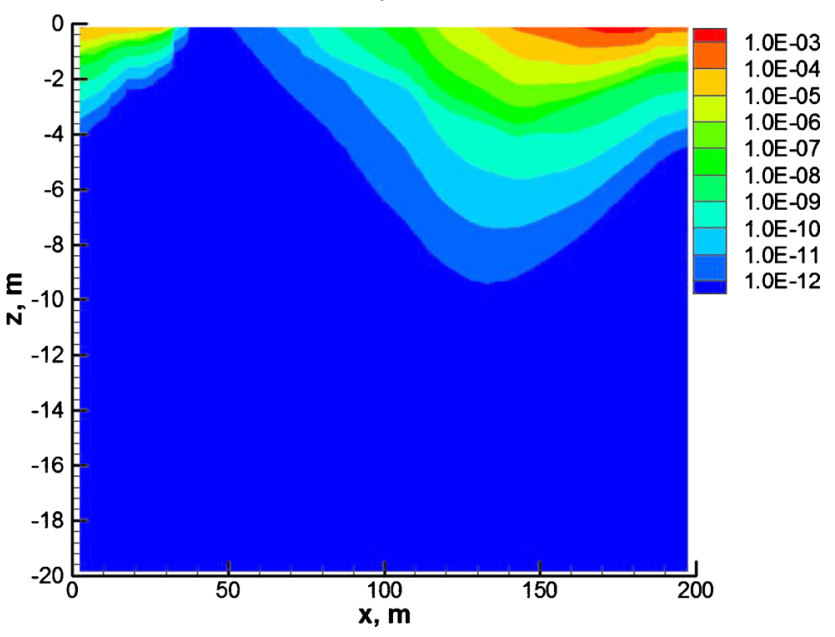

FIG. 7. (Color online) (a) Acoustic speed (meters per second) and (b) void fraction of bubbles $\left(1 / \mathrm{m}^{3}\right)$ for periodic internal wave of $3 \mathrm{~m}$ amplitude and wind speed $W=2.5 \mathrm{~m} / \mathrm{s}$ (structure due to inhomogeneous breaking). 


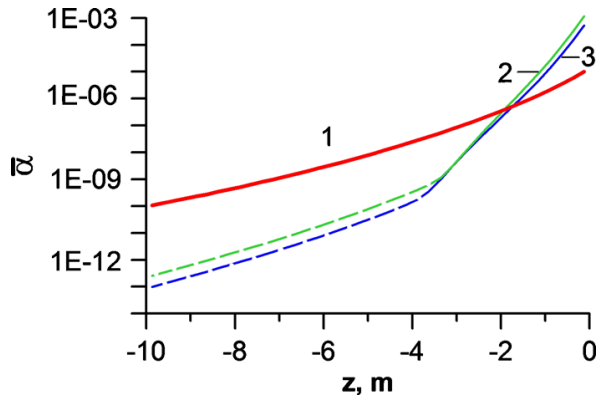

FIG. 8. (Color online) Averaged void fraction as a function of depth. (1) $W=10 \mathrm{~m} / \mathrm{s}$ (homogeneous input of bubbles), (2) $W=2.5 \mathrm{~m} / \mathrm{s}$ [inhomogeneous input of bubbles according to Eq. (21)], and (3) $W=2.5 \mathrm{~m} / \mathrm{s}$ [inhomogeneous input of bubbles according to Eq. (22)]. The dashed line effectively corresponds to the absence of bubbles (radius of bubbles is less than $5 \mu \mathrm{m})$.

of counterpropagating internal and surface waves and hence we expect surface wave breaking to be not so prominent.

\section{CONCLUDING REMARKS}

In this paper we have modeled the shaping of the subsurface bubble layer by an internal wave. The effect of an internal wave on the surface wave breaking and, thus, on the subsurface bubble layer, was first observed in Ref. 18. Recently, structure formation in the bubble layer in accordance with the profile of the underlying internal wave was recorded on the shelf of Sea of Japan in Refs. 19 and 20.

We considered an uncoupled problem formulation using the solution for an internal wave in a two-layer fluid, which was then substituted into the equation describing the bubble dynamics. We studied two different cases, when breaking is caused either by a strong wind or by the interaction with an internal wave. We have proposed a simple analytical model for the injection of bubbles through the surface due to the modulation and breaking of surface waves induced by an internal wave. The equations were solved numerically, showing structure formation in the bubble layer.

The mathematical model used in our study for the very complex processes in the turbulent ocean is highly idealized. In particular, in the case when breaking is caused by a strong sustained wind, it is desirable to try to develop this study further to account for the effects of Langmuir circulation, which also plays a significant role in the dynamics of bubbles (see Refs. 32-34). In the case when surface waves breaking is caused by a direct interaction with an internal wave, it is interesting to consider an oblique interaction. For simplicity, in the current study we used a solution for a linear sinusoidal internal wave. However, large amplitude nonlinear internal waves are commonly observed in the coastal oceans (e.g., Ref. 23 and references therein) and one could consider the organization of bubbles by such nonlinear waves. The theoretical framework developed in this paper can be generalized to account for these extensions.

The described effects are twofold: bubbles are driven by an internal wave field and the injection of bubbles into the water is enhanced due to the steepening of surface waves by the internal wave. We have shown that both of these mechanisms increase the void concentration of bubbles in the downwelling areas behind the crests of the internal wave, which agree with the observations in Refs. 18-20.

We also calculated the characteristics such as bubble void fraction, acoustic wave speed in the mixture, and scattering intensity, and showed that the structure formation in the bubble layer can have a strong effect on the acoustics in the subsurface layer. We have shown that the horizontal variability of these characteristics approximately copies the internal wave, with a shift in the direction of the propagation of the internal wave. The magnitude of such variability is depth-dependent.

Thus, processes of this type should be important for the study of underwater acoustics and the related oceanographic measurement techniques based on acoustic Doppler current profilers (ADCPs).

\section{ACKNOWLEDGMENTS}

We thank A. N. Serebryany for Refs. 19 and 20 and D. M. Farmer for Ref. 21. R. H. J. Grimshaw and K. R. Khusnutdinova thank the Royal Society and Department of Mathematical Sciences of Loughborough University for financial support of A. S. Topolnikov to Loughborough. K. R. Khusnutdinova thanks the London Mathematical Society and Science Faculty of Loughborough University for financial support of L. A. Ostrovsky to Loughborough.
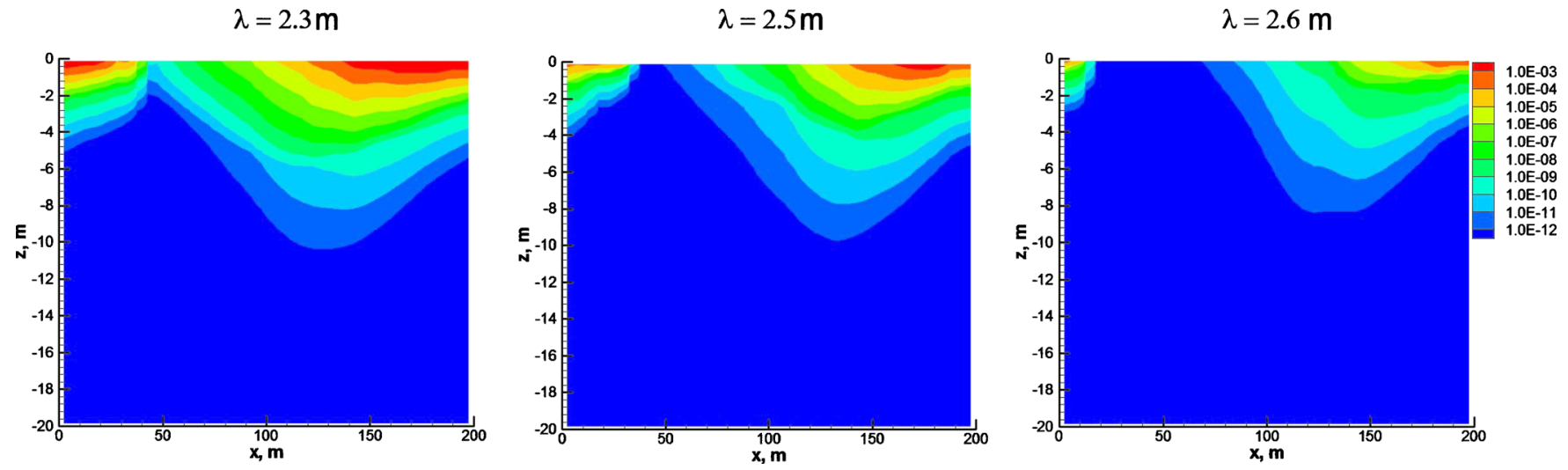

FIG. 9. (Color online) The void fraction for different wavelengths of surface waves: $2.3,2.5$, and $2.6 \mathrm{~m}$. The amplitude is $a_{s 0}=0.1 \mathrm{~m}$. 

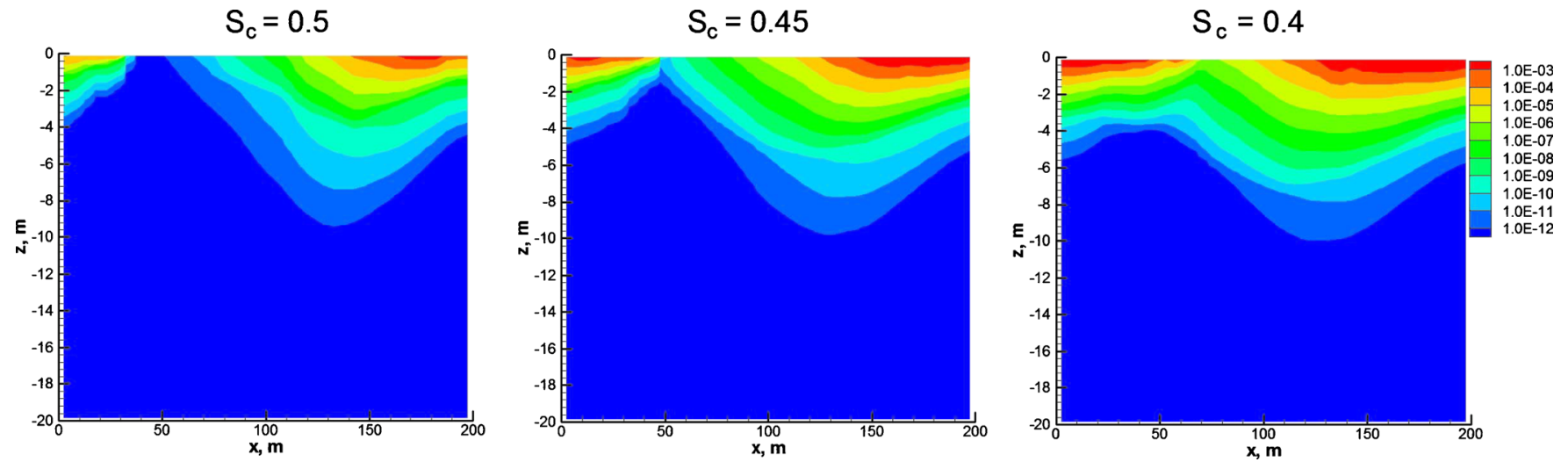

FIG. 10. (Color online) The void fraction for different values of the critical steepness: $0.5,0.45$, and 0.4 . Here, $\lambda_{0}=3.0 \mathrm{~m}$ and $a_{s 0}=0.1 \mathrm{~m}$.

\section{APPENDIX A: INTERNAL WAVES IN A TWO-LAYER FLUID}

In this appendix, we present a brief summary of the well-known solution for a linear internal wave, summarizing all necessary formulae (e.g., Refs. 26-28). We suppose that the flow is two-dimensional and use the spatial coordinates $(x, z)$, where $x$ is horizontal and $z$ is vertical. The undisturbed free surface is at $z=0$. The upper layer has an undisturbed constant depth $h$ and a constant density $\rho_{1}$, while the lower layer has an undisturbed constant depth $H-h$ and a constant density $\rho_{2}$ ( $H$ is the total depth). We consider an inviscid, incompressible fluid.

Let $u_{i}, w_{i}$ denote the horizontal and vertical components of the velocity field, $p_{i}$ be the pressure deviation from the hydrostatic pressure, and $\rho_{i}$ be the density in each layer ( 1 and 2 denote the upper and lower layers, respectively). The linear internal wave field is then easily found by solving the linearized Euler equations in each layer

$$
\rho_{i} u_{i_{t}}+p_{i_{x}}=0, \quad \rho_{i} w_{i_{t}}+p_{i_{z}}+\rho_{i} g=0, \quad u_{i_{x}}+w_{i_{z}}=0
$$

subject to the usual rigid lid condition at the surface

$$
\left.w_{1}\right|_{z=0}=0
$$

rigid bottom condition at $z=-H$

$$
\left.w_{2}\right|_{z=-H}=0 \text {, }
$$

and linearized continuity conditions for the vertical velocity and pressure at the interface

$$
\begin{aligned}
& \left.w_{1}\right|_{z=-h}=\left.w_{2}\right|_{z=-h}=\zeta_{t}, \\
& p_{1}-\left.p_{2}\right|_{z=-h}=-\left(\rho_{2}-\rho_{1}\right) g \zeta,
\end{aligned}
$$

where $\zeta$ denotes the elevation of the interface from the undisturbed level $z=-h$. Assuming that $\rho_{2}-\rho_{1} \ll \rho_{1}, \rho_{2}$, and denoting $\Delta \rho=\left(\rho_{2}-\rho_{1}\right) / \rho_{2}$, Eq. (A4) yields an interface condition in the form

$$
w_{1_{z t}}-\left.w_{2_{z t}}\right|_{z=-h}=-\Delta \rho g \zeta_{x x} .
$$

Then the solution is found by solving the Laplace equation in each layer

$$
w_{i_{x x}}+w_{i_{z z}}=0, \quad i=1,2,
$$

subject to the conditions (A1)-(A3) and (A5).

Next, let

$$
\zeta(x, t)=A e^{i(k x-\omega t)}+c . c .
$$

and then we find that the internal wave field in the upper layer is given by

$$
\begin{aligned}
& u_{1}=-\frac{\omega A}{\sinh k h} \cosh k z e^{i(k x-\omega t)}+c . c ., \\
& w_{1}=\frac{i \omega A}{\sinh k h} \sinh k z e^{i(k x-\omega t)}+c . c ., \\
& p_{1}=-\rho_{1} \frac{\omega^{2} A}{k \sinh k h} \cosh k z e^{i(k x-\omega t)}+c . c . .
\end{aligned}
$$

The total pressure in the upper layer is

$$
p_{1}^{\text {total }}=p_{a}-\rho_{1} g z+p_{1},
$$

where $p_{a}$ is the atmospheric pressure. The linearized internal wave field in the lower layer is given by

$$
\begin{aligned}
& u_{2}=\frac{\omega A}{\sinh k(H-h)} \cosh k(z+H) e^{i(k x-\omega t)}+c . c ., \\
& w_{2}=-\frac{i \omega A}{\sinh k(H-h)} \sinh k(z+H) e^{i(k x-\omega t)}+c . c ., \\
& p_{2}=\rho_{2} \frac{\omega^{2} A}{k \sinh k(H-h)} \cosh k(z+H) e^{i(k x-\omega t)}+c . c . .
\end{aligned}
$$

The total pressure in the lower layer is

$$
p_{2}^{\text {total }}=p_{a}+\rho_{1} g h-\rho_{2} g(z+h)+p_{2} .
$$

The dispersion relation is given as follows:

$$
\omega^{2}=\Delta \rho g k \frac{\tanh k h \cdot \tanh k(H-h)}{\tanh k(H-h)+\tanh k h} .
$$




\section{APPENDIX B: MODULATION OF SURFACE WAVES BY AN INTERNAL WAVE}

The modulation of surface waves by a background current was studied in Refs. 35-38 These theories were adapted to the modulation of surface waves by an internal wave (see, for instance, Refs. 39 and 40). An alternative model commonly used for the interpretation of images of the surface signature of internal waves was introduced in Ref. 41 under the assumptions that the surface wave field is maintained by a strong wind and the internal wave is regarded as a perturbation to this field. Here we present a brief summary of these theories and present the main results needed for our present study. In particular, we will emphasize the distinction between a fixed reference frame (that used by an observer at a fixed place) and the reference frame, which moves with the internal wave and in which the internal wave is steady.

First, consider the Euler equations in the fixed reference frame. Using $x, z, t$ coordinates, the horizontal momentum equation is

$$
u_{t}+u u_{x}+w u_{z}+p_{x}=0 .
$$

We are concerned with surface waves, with a horizontal velocity field $u_{s}$, riding on the surface current of an internal wave $u_{i}=u_{i}\left(x-c_{i} t, z\right)$. Linearization then yields

$$
u=u_{i}+u_{s}, \quad u_{s t}+u_{i} u_{s x}+\left[u_{s} u_{i x}+w_{i} u_{s z}+w_{s} u_{i z}\right]+p_{s z}=0 .
$$

On the assumption that the internal wave is a long wave and slowly varying relative to the surface wave, the terms $[\cdots]$ can be neglected at the leading order, but this is not essential at the moment.

Then to facilitate the analysis, we make a change of variables

$$
X=x-c_{i} t, T=t, Z=z, U=u, W=w, P=p,
$$

so that Eq. (B1) becomes

$$
u_{T}+\left(u-c_{i}\right) u_{X}+w u_{Z}+p_{X}=0
$$

and Eq. (B2) becomes

$$
u_{s T}+\left(u_{i}-c_{i}\right) u_{s X}+\left[u_{s} u_{i X}+w_{i} u_{s Z}+w_{s} u_{i Z}\right]+p_{s Z}=0 .
$$

In effect, the internal wave current $u_{i}\left(x-c_{i} t, z\right)$ becomes $u_{i}(X, Z)-c_{i}$. Alternatively, we can make a change of variables, preserving Galilean invariance

$$
X=x-c_{i} t, T=t, Z=z, U=u-c_{i}, W=w, P=p,
$$

so that

$$
U_{T}+U U_{X}+W U_{Z}+P_{X}=0 .
$$

But now, the linearization is about $U_{i}(X, Z)=u_{i}(X, Z)-c_{i}$, that is $U=U_{i}+u_{s}$ and so again we get Eq. (B3). That is, both transformations lead to the same equation for the calculation of the modulated surface wave field.

Now we can apply a WKB-type analysis to the modulation of surface waves by slowly varying current (see, for instance, Refs. 38, 40, 43, and 44). The outcome is that the dispersion relation is the same as when the internal wave is omitted, except for the Doppler shift by the surface current
$V(X)=u_{i}(X, 0)-c_{i}$, and then the equation for conservation of waves determines how the surface wave number will be modulated. The surface wave amplitude is governed by the wave action equation. The fact that only $u_{i}(X, 0)$ is needed requires some careful work, but is essentially the outcome of the long-wave hypothesis for the internal wave and the assumption that the internal wave is slowly varying relative to the surface wave. Thus, in the comoving frame, a modulated deep-water surface wave of elevation $\zeta_{s}$ is described by

$$
\zeta_{s}=a_{s} \cos \phi, \quad \phi_{T}=-\Omega, \quad \phi_{X}=\kappa,
$$

where the frequency $\Omega$, wave number $\kappa$, and amplitude $a_{s}$ are slowly varying functions of $X, T$ determined by the equations

$$
\begin{aligned}
& \Omega=V(X)_{\kappa}+\Omega^{*}, \quad \Omega^{* 2}=g|\kappa|, \\
& \kappa_{T}+\Omega_{X}=0, \\
& \mathbf{A}_{T}+\left(c_{g} \mathbf{A}\right)_{X}=0, \quad \mathbf{A}=\frac{E}{\Omega^{*}}, \quad E=\frac{1}{2} \rho_{l} g a_{s}^{2}, \quad c_{g}=\frac{\partial \Omega}{\partial \kappa} .
\end{aligned}
$$

Here, $\mathbf{A}$ is the wave action density and $E$ is the wave energy density. These equations have the steady solution in which $\Omega$ is a constant and $\kappa, a_{s}$ depend only on $X$

$$
\Omega=\Omega_{0}, \quad \phi=\int_{X_{0}}^{X} \kappa\left(X^{\prime}\right) d X^{\prime}-\Omega_{0} T, \quad \frac{\rho_{l} c_{g} a_{s}^{2}}{2 \Omega^{*}}=F_{0},
$$

where $\Omega_{0}, F_{0}$ are constants. The dispersion relation then provides a prescription for the dependence of $\kappa=\kappa(V)$, which is better expressed in terms of the intrinsic phase speed $c^{*}=\Omega^{*} / \kappa$

$$
c^{* 2}=c_{0}\left[V(X)+c^{*}\right], \quad c_{0}=\frac{g}{\Omega_{0}} .
$$

Here, without loss of generality we have chosen $\kappa>0$. The solution for $c^{*}, \kappa, c_{g}$ is

$$
c^{*}=\frac{c_{0}}{2} \pm c_{0}\left[\frac{V(X)}{c_{0}}+\frac{1}{4}\right]^{1 / 2}, \quad \frac{\kappa}{\kappa_{0}}=\frac{c_{0}^{2}}{c^{* 2}},
$$

$$
c_{g}=V(X)+\frac{c^{*}}{2}=V(x)+\frac{c_{0}}{4} \pm \frac{c_{0}}{2}\left[\frac{V(X)}{c_{0}}+\frac{1}{4}\right]^{1 / 2} .
$$

Now choose $c_{0}$ to be $c^{*}(V=0)$, so that the plus sign is chosen and again, without loss of generality, we can choose $c_{0}>0$, so that the surface waves propagate in the positive $x$-direction. For $V>0$ the surface waves propagate without restriction and $c^{*}$ decreases as $V$ increases, while for $V<0$, $c^{*}$ increases to infinity as $V$ decreases to the stopping velocity of $-c_{0} / 4$. The wave amplitude is given by Eq. (B8) which reduces to 


$$
\frac{a_{s}^{2}(X)}{a_{s 0}^{2}}=\frac{1}{2}\left[\frac{V(X)}{c_{0}}+\frac{1}{4}\right]^{-1 / 2}\left\{\frac{1}{2}+\left[\frac{V(X)}{c_{0}}+\frac{1}{4}\right]^{1 / 2}\right\}^{-2} .
$$

For $V$ positive and increasing, the surface wave energy $a_{s}^{2}$ decreases, while for $V$ negative and decreasing, $a_{s}^{2}$ increases to infinity at the stopping velocity. In this case wave breaking will occur before the stopping velocity.

This well-known solution (B11) for the modulation of surface waves by a slowly varying current assumes that the surface wave field is essentially unforced. In the situation when there are strong winds, this assumption needs to be relaxed. Following Ref. 40, we assume that there is a dominant wave number in the wind-wave field, the dispersion relation still holds, and so Eqs. (B5) and (B6) can again be used to yield the expressions (B9) and (B10). But the wave action equation is changed by the presence of source and dissipation terms. Thus, in the comoving frame, the wave action Eq. (B7) is changed to

$$
\mathbf{A}_{T}+\left(c_{g} \mathbf{A}\right)_{X}=S-D, \quad \mathbf{A}=\frac{\rho_{l} g a_{s}^{2}}{2 \Omega^{*}}, \quad c_{g}=\frac{\partial \Omega}{\partial \kappa} .
$$

Here $S$ is a source term, and $D$ is a "dissipation" term representing nonlinear interactions and energy loss to small scales (possibly through wave breaking). The solution of this equation now requires a more detailed knowledge of these source and dissipation terms. However, we can follow an approach pioneered in Ref. 41 in which Eq. (B12) is used to determine $\mathbf{A}=\mathbf{A}(\kappa)$. First assume that there is steady state in which $\kappa=\kappa_{0}$ (a constant), $\mathbf{A}=\mathbf{A}_{0}=\mathbf{A}\left(\kappa_{0}\right)$ so that $S=D$. Then suppose that this steady state is perturbed by the effect of the internal wave surface current $V(X)=u_{i}(X, 0)-c_{i}$. Then $\kappa=\kappa_{0}+\delta \kappa, \mathbf{A}=\mathbf{A}_{0}(\kappa)+\delta \mathbf{A}$ is the action density of the perturbed state, and following Ref. 41, we assume the perturbation of $S-D$ to be $-\mu \delta \mathbf{A}$. Here $\mu$ is an empirically determined constant, such that $\mu^{-1}$ is typically of the order 10 100 wave periods. The perturbed wave kinematic Eqs. (B5) and (B6) yield the outcome

$$
\frac{\delta \kappa}{\kappa_{0}}=-\frac{V(X)}{c_{g 0}}, \quad \text { since } \quad \Omega=V(X) \kappa+\Omega^{*}(\kappa)=\Omega_{0},
$$

while the perturbed action spectrum is given by

$$
\delta \mathbf{A}_{T}+c_{g 0} \delta \mathbf{A}_{X}+\left(1-\frac{\kappa_{0}}{c_{g 0}} \frac{\partial c_{g 0}}{\partial \kappa_{0}}-\frac{\kappa_{0}}{\mathbf{A}_{0}} \frac{\partial \mathbf{A}_{0}}{\partial \kappa_{0}}\right) \mathbf{A}_{0} V_{X}=-\mu \delta \mathbf{A} .
$$

Here the subscript 0 refers to the steady state without the modulations induced by the internal wave and we note that $\kappa_{0} \partial c_{g 0} / \partial \kappa_{0}=-c_{g 0} / 2$. Following Ref. 41, we assume that the relaxation term on the right-hand side dominates over the local term $\delta \mathbf{A}_{T}+c_{g 0} \delta \mathbf{A}_{X}$, which then yields the quasisteady state solution

$$
\frac{\delta \mathbf{A}}{\mathbf{A}_{0}}=-\frac{(3+2 \beta)}{2 \mu} V_{X}, \quad \beta=-\frac{\kappa_{0}}{\mathbf{A}_{0}} \frac{\partial \mathbf{A}_{0}}{\partial \kappa_{0}},
$$

which is similar to the formula obtained in Ref. 41. For a spectrum $E \sim \kappa^{-4}$, or $A \sim \kappa^{-9 / 2}, \beta=9 / 2$, and $3 / 2+\beta=6$.
Next we note that the corresponding action change due to the modulation of the surface wave directly by the internal wave current $V(X)$ in the absence of any wind is expressed by Eqs. (B7) and (B8), which for small values of $V(X)$ yields to leading order

$$
\frac{\delta \mathbf{A}}{\mathbf{A}_{0}}=-3 \frac{V(X) \kappa_{0}}{\Omega_{0}} .
$$

Note that expression (B13) peaks at the downwelling center, while expression (B14) peaks over the crest of the internal wave, in the absence of any wave breaking. For the interfacial wave described in Appendix A, the ratio of these terms can be estimated in magnitude as $(3+2 \beta) k \Omega_{0} / 6 \kappa_{0} \mu$, where $k$ is the wave number of the interfacial wave. In the oceanic situation, $k \ll \kappa_{0}$ but $\Omega_{0} \gg 2 \pi \mu$, and so which term dominates depends on the actual case being considered. A typical scenario might have $k / \kappa_{0} \sim 10^{-2}$, while $\Omega_{0} / 2 \pi \mu$ varies in the range 10-100; then the ratio varies in the range 1-10 in order of magnitude, suggesting that usually it will be the first term which dominates. However, we must recall that expression (B13) is derived on the assumption that the internal wave field is quite weak $\left(V \ll c_{0}\right)$ and for stronger internal wave fields, we would expect expression (B11) derived for unforced surface wave fields to hold.

${ }^{1}$ E. C. Monahan and I. O'Muircheartaigh, "Optimal power-law description of oceanic whitecap coverage dependence on wind speed," J. Phys. Oceanogr. 10, 2094 (1980).

${ }^{2} \mathrm{~S}$. A. Thorpe, "On the clouds of bubbles formed by breaking wind-waves in deep water, and their role in air-sea gas transfer," Philos. Trans. R. Soc. London, Ser. A 304, 155 (1982).

${ }^{3}$ D. M. Farmer and S. Vagle, "Waveguide propagation of ambient sound in the ocean-surface bubble layer," J. Acoust. Soc. Am. 86, 1897 (1989).

${ }^{4}$ L. Zedel and D. Farmer, "Organized structures in subsurface bubble clouds: Langmuir circulation in the open ocean," J. Geophys. Res. 96, 8889, doi:10.1029/91JC00189 (1991).

${ }^{5}$ D. M. Farmer, S. Vagle, and M. Li, "Wave breaking, turbulence and bubble distributions in the ocean surface layer," in Proceedings of the Symposium on the Wind-Driven Air-Sea Interface, Sydney, Australia, 11-15 January 1999, edited by M. L. Banner (The University of New South Wales, Sydney, 1999), pp. 187-192.

${ }^{6}$ D. M. Farmer and D. D. Lemon, "The influence of bubbles on ambient noise in the ocean at high wind speeds," J. Phys. Oceanogr. 14, 1762 (1984).

${ }^{7}$ M. J. Buckingham, "Sound speed and void fraction profiles in the sea surface bubble layer," Appl. Acoust. 51, 225 (1997).

${ }^{8} \mathrm{~S}$. A. Thorpe, "A model of the turbulent diffusion of bubbles below the sea surface," J. Phys. Oceanogr. 14, 841 (1984).

${ }^{9} \mathrm{~S}$. A. Thorpe, "On the determination of $K_{v}$ in the near-surface ocean from acoustic measurements of bubbles," J. Phys. Oceanogr. 14, 855 (1984).

${ }^{10}$ E. J. Terrill and W. K. Melville, "Field measurements of bubble size distributions in the upper mixed layer," in Proceedings of the Symposium on the Wind-Driven Air-Sea Interface, Sydney, Australia, 11-15 January 1999, edited by M. L. Banner (The University of New South Wales, Sydney, 1999), pp. 247-255.

${ }^{11}$ S. V. Iordansky, "Equations of motion of liquid containing gas bubbles," Zh. Tekh. Fiz. 3, 102 (1960).

${ }^{12}$ B. S. Kogarko, "On the model of cavitating liquid," Dokl. Akad. Nauk SSSR 137, 1331 (1961).

${ }^{13} \mathrm{~L}$. Van Wijngaarden, "On the equations of motion for mixtures of liquid and gas bubbles," J. Fluid Mech. 33, 465 (1968).

${ }^{14}$ R. I. Nigmatulin, Dynamics of Multiphase Media (Hemisphere, Washington, D.C., 1991), Vols.1-2.

${ }^{15}$ R. H. J. Grimshaw and K. R. Khusnutdinova, "The effect of bubbles on internal waves," J. Phys. Oceanogr. 34, 477 (2004). 
${ }^{16}$ R. H. J. Grimshaw and K. R. Khusnutdinova, "Internal waves in a threelayer bubbly waveguide," Deep-Sea Res., Part II 51, 2905 (2004).

${ }^{17}$ R. H. J. Grimshaw, K. R. Khusnutdinova, and L. A. Ostrovsky, "The effect of a depth-dependent bubble distribution on the normal modes of internal waves: Quasistatic approximation,” Eur. J. Mech. B/Fluids 27, 24 (2008).

${ }^{18}$ S. A. Thorpe, M. B. Belloul, and A. J. Hall, "Internal waves and whitecaps," Nature (London) 330, 740 (1987).

${ }^{19}$ A. N. Serebryany and N. N. Galybin, "Effect of internal wave on subsurface layer of air bubbles in the sea," Proceedings of the 11th L. M. Brekhovskikh Conference on Ocean Acoustics, Moscow, Russia, 23-26 May 2006 (Russian Academy of Sciences, Moscow, 2006), pp. 378-382 (in Russian).

${ }^{20}$ A. Serebryany and N. Galybin, "Observations of the effect of internal waves on subsurface layer of air bubbles in the sea," Proceedings of the Third International Conference on Underwater Acoustic Measurements: Technologies and Results (Third UAM), Nafplion, Greece, 22-26 June 2009, edited by J. Papadakis and L. Bjorno, pp. 775-780.

${ }^{21}$ B. Baschek, D. M. Farmer, and C. Garrett, "Tidal fronts and their role in air-sea gas exchange,” J. Mar. Res. 64, 483 (2006).

${ }^{22}$ L. A. Ostrovsky and J. Grue, "Evolution equations for strongly nonlinear internal waves," Phys. Fluids 15, 2934 (2003).

${ }^{23}$ K. R. Helfrich and W. K. Melville, "Long nonlinear internal waves," Annu. Rev. Fluid Mech. 38, 395 (2006).

${ }^{24}$ C. Garrett and E. Kunze, "Internal tide generation in the deep ocean," Annu. Rev. Fluid Mech. 39, 57 (2007).

${ }^{25}$ T. Peacock, M. J. Mercier, H. Didelle, S. Viboud, and T. Dauxois, "A laboratory study of low-mode internal tide scattering by finite-amplitude topography," Phys. Fluids 21, 121702 (2009).

${ }^{26} \mathrm{P}$. H. LeBlond and L. A. Mysak, Waves in the Ocean (Elsevier, Amsterdam, 1978).

${ }^{27}$ A. E. Gill, Atmosphere-Ocean Dynamics (Academic, New York, 1982).

${ }^{28}$ Yu. Z. Miropol'sky, Dynamics of Internal Gravity Waves in the Ocean (Kluwer, Dordrecht, 2001).
${ }^{29}$ S. V. Patankar, Numerical Heat Transfer and Fluid Flow (Hemisphere, Washington, D.C., 1980).

${ }^{30} \mathrm{M}$. V. Hall, "A comprehensive model of wind-generated bubbles in the ocean and predictions of the effects on sound propagation at frequencies up to $40 \mathrm{kHz}$," J. Acoust. Soc. Am. 86, 1103 (1989).

${ }^{31}$ S. Thorpe and A. Stubbs, "Bubbles in a freshwater lake," Nature (London) 279, 403 (1979).

${ }^{32}$ D. M. Farmer and M. Li, "Patterns of bubble clouds organised by Langmuir circulation," J. Phys. Oceanogr. 25, 1426 (1995).

${ }^{33} \mathrm{M}$. Li and C. Garrett, "Mixed-layer deepening due to Langmuir circulation," J. Phys. Oceanogr. 27, 121 (1997).

${ }^{34}$ S. A. Thorpe, "Langmuir circulation," Annu. Rev. Fluid Mech. 36, 55 (2004).

${ }^{35}$ M. S. Longuet-Higgins and R. W. Stewart, "The changes in amplitude of short gravity waves on steady nonuniform currents," J. Fluid Mech. 10, 529 (1961).

${ }^{36}$ G. B. Whitham, "Mass, momentum and energy flux in water waves," J. Fluid Mech. 12, 135 (1962).

${ }^{37}$ O. M. Phillips, The Dynamics of the Upper Ocean (Cambridge University Press, Cambridge, 1966).

${ }^{38}$ F. P. Bretherton and C. J. R. Garrett, "Wavetrains in inhomogeneous moving media," Proc. R. Soc. London, Ser. A 302, 529 (1968).

${ }^{39}$ A. E. Gargett and B. A. Hughes, "On the interaction of surface and internal waves," J. Fluid Mech. 52, 179 (1972).

${ }^{40}$ A. N. Donato, D. H. Peregrine, and J. R. Stocker, "The focusing of surface waves in internal waves," J. Fluid Mech. 384, 27 (1999).

${ }^{41}$ W. Alpers, "Theory of radar imaging of internal waves," Nature (London) 314, 245 (1985).

${ }^{42}$ C. C. Mei, The Applied Dynamics of Ocean Surface Waves (Wiley, New York, 1983).

${ }^{43}$ G. B. Whitham, Linear and Nonlinear Waves (Wiley, New York, 1974).

${ }^{44} \mathrm{R}$. S. Johnson, A Modern Introduction to the Mathematical Theory of Water Waves (Cambridge University Press, Cambridge, 1997). 